\title{
Oxygen abundances in G- and F-type stars from HARPS ${ }^{\star}$ Comparison of [OI] $6300 \AA$ and OI $6158 \AA$
}

\author{
S. Bertran de Lis ${ }^{1,2}$, E. Delgado Mena ${ }^{3}$, V. Zh. Adibekyan ${ }^{3}$, N. C. Santos ${ }^{3,4}$, and S. G. Sousa ${ }^{1,3,4}$ \\ ${ }^{1}$ Instituto de Astrofísica de Canarias, 38205 La Laguna, Tenerife, Spain \\ e-mail: sbertran@iac.es \\ 2 Universidad de La Laguna, Dept. Astrofísica, 38206 La Laguna, Tenerife, Spain \\ 3 Centro de Astrofísica da Universidade do Porto, Rua das Estrelas, 4150-762 Porto, Portugal \\ 4 Departamento de Física e Astronomia, Faculdade de Ciências da Universidade do Porto, 4150-762 Porto, Portugal
}

Received 18 July 2014 / Accepted 6 January 2015

\begin{abstract}
Aims. We present a detailed and uniform study of oxygen abundance from two different oxygen lines at $6158 \AA$ and $6300 \AA$ in a large sample of solar-type stars. The results are used to check the behaviour of these spectral lines as oxygen abundance indicators and to study the evolution of oxygen in thick and thin disk populations of the Galaxy.

Methods. Equivalent width measurements were carried out for the [OI] $6158 \AA$ and OI $6300 \AA$ lines. Local thermodynamic equilibrium (LTE) abundances were obtained from these two lines in 610 and 535 stars, respectively. We were able to measure oxygen abundance from both indicators in 447 stars enabling us for the first time to compare them in a uniform way. Careful error analysis has been performed.

Results. We found that oxygen abundances derived from the $6158 \AA$ and $6300 \AA$ lines agree to within 0.1 dex in $58 \%$ of the stars in our sample, and this result improves for higher signal-to-noise values. We confirm an oxygen enhancement in stars of the thick disk, as has also been seen for other $\alpha$-elements. The new oxygen abundances confirm previous findings for a progressive linear rise in the oxygen-to-iron ratio with a slope equal to 0.78 from solar metallicity to $[\mathrm{Fe} / \mathrm{H}] \sim-1$. However, the slope we measured is steeper than the one found in previous studies based on the oxygen triplet. Below $[\mathrm{Fe} / \mathrm{H}]=-0.6$ our stars show $[\mathrm{O} / \mathrm{Fe}]$ ratios as high as $\sim 0.8$, which can be interpreted as evidence for oxygen overproduction in the Galactic thick disk. These high oxygen abundances do not pose a problem to chemodynamical models since there is a range of parameters that can accommodate our results.
\end{abstract}

Key words. stars: abundances - stars: atmospheres - stars: solar-type - Galaxy: abundances

\section{Introduction}

After hydrogen and helium, oxygen is the most abundant element in the Universe. As a matter of fact, oxygen is the only chemical element that has a unique production site. This has been used by many authors to argue that oxygen is the best tracer of galactic chemical evolution. It is produced by alpha particles during hydrostatic nucleosynthesis in massive stars. The interstellar medium is enriched with oxygen when massive stars explode as Type II supernovae. (e.g. Wheeler et al. 1989; Maeder 2009; Stasińska et al. 2012).

Despite the importance of deriving precise oxygen abundances in solar-type stars, this is usually not a trivial process. The number of spectral lines of atomic oxygen suitable for abundance analysis in visual spectra is small. There is a large number of molecular lines of $\mathrm{OH}$ in the near-UV and near-IR, strong enough to be measured in main-sequence metal-poor stars down to $[\mathrm{Fe} / \mathrm{H}] \sim-3.5$ dex (Israelian et al. 1998, 2001; Boesgaard et al. 1999). However, it is extremely difficult to measure abundances from molecular features as they are affected by several physical processes that are not well understood in solar-type

* Full Tables 2, 3, A.1, and A.2 are only available at the CDS via anonymous ftp to cdsarc.u-strasbg. fr $(130.79 .128 .5)$ or via http://cdsarc.u-strasbg.fr/viz-bin/qcat?]/A+A/576/A89 stars (UV opacities, etc.). The situation with atomic lines is no more promising. Serious disagreements exist in both solartype main sequence and evolved sub-giant/giant stars in any metallicity range. The well-known spectral lines of atomic oxygen suitable for abundance analysis are [OI] $6300 \AA$ and the OI triplet at 7771-4 $\AA$. The weak spectral lines of OI $6158 \AA$ and [OI] $6363 \AA$ are hardly used owing to their small equivalent widths (EWs). The strong lines of the oxygen triplet are strongly affected by deviations from local thermodynamic equilibrium (LTE; e.g. Caffau et al. 2008), which depend on the cross section of collisions between the atoms of oxygen and neutral hydrogen. The physical description of these collisions is still a matter of scientific debate. Moreover, the forbidden line is often blended with telluric features and also contains a $\mathrm{Ni}$ I blend at 6300.40 A (e.g. Lambert 1978; Allende Prieto et al. 2001) and a much weaker CN blend (e.g. Teske et al. 2013). The Ni line, with an uncertain oscillator strength, may contribute up to $60 \%$ to the EW of the $6300 \AA$ feature (see Sect. 4). In addition, a disagreement between the OI triplet and forbidden line abundances in metal-poor giants can reach up to 1 dex (Israelian et al. 2004).

There are many articles dedicated to oxygen measurements in solar-type stars. Several workshops and conferences have been held to review the status of oxygen measurements and address unresolved problems related to inconsistent abundances 

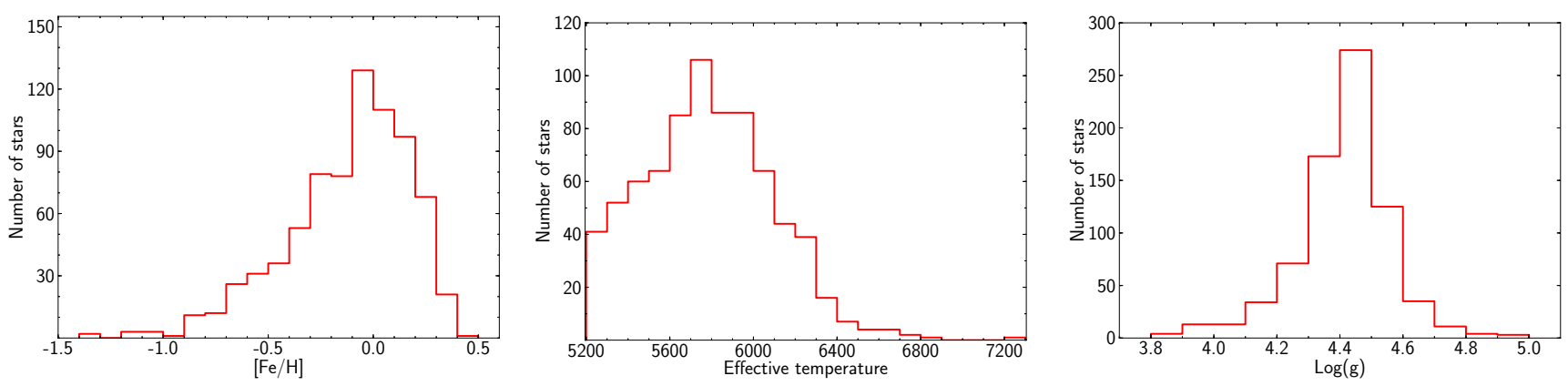

Fig. 1. Distribution of the fundamental stellar parameters in the HARPS sample for stars with effective temperatures higher than $5200 \mathrm{~K}$.

derived from different oxygen lines. Most of the previous work on oxygen with large samples of stars (e.g. Bensby et al. 2004; Takeda \& Honda 2005; Ecuvillon et al. 2006; Delgado Mena et al. 2010; Petigura \& Marcy 2011; Ramírez et al. 2013) were based on two lines: OI triplet $\sim 7774 \AA$ and [OI] $6300 \AA$. Ecuvillon et al. (2006) have compared near-UV OH lines with the OI triplet and $[\mathrm{OI}]$ and found good agreement between the $[\mathrm{O} / \mathrm{H}]$ ratios from forbidden and $\mathrm{OH}$ lines, while the nonLTE triplet showed a systematically lower abundance. Takeda \& Honda (2005) have used the $6158 \AA$ OI line to derive oxygen abundances in 160 FGK dwarfs and sub-giants. Nevertheless, the quality of their data was not high enough to reach conclusions about the reliability of this line.

To all the above-mentioned we must add that the solar oxygen abundance itself is still uncertain. Since the first determinations of Lambert $(1978)(\log \epsilon(\mathrm{O})=8.92)$ and Anders \& Grevesse (1989) $(\log \epsilon(\mathrm{O})=8.93)$, the value of solar oxygen has undergone several revisions that yielded progressively lower values. In recent years Asplund et al. (2004) have recommended a value of $\log \epsilon(\mathrm{O})=8.66 \pm 0.05$ based on measurements of [OI], $\mathrm{OI}$, and IR OH lines, which was supported by the Socas-Navarro $\&$ Norton $(2007)$ determination $(\log \epsilon(\mathrm{O})=8.63)$ using the infrared triplet. This value was reviewed in 2009 to $\log \epsilon(\mathrm{O})=$ $8.69 \pm 0.05$ using new 3D hydrodynamical models of the solar atmosphere (Asplund et al. 2009). On the other hand Ayres (2008) determined a significantly larger value $(\log \epsilon(\mathrm{O})=8.81)$ based on a single snapshot of a 3D model for the [OI]6300 line and treating the $\log (g f)$ of Ni blended line as a free parameter. Recently, Caffau et al. (2008) presented a new determination of the solar photospheric oxygen abundance by analysing different spectral atlases of the solar flux and disk-centre intensity, making use of the latest generation of CO5BOLD 3D solar model atmospheres. They have studied the photospheric oxygen abundance by considering only lines from atomic transitions. Ignoring the role of collisions with hydrogen atoms on the non-LTE level populations of oxygen, they proposed $\log \epsilon(\mathrm{O})=8.76 \pm 0.07$. They have stressed that the measurement of EWs with high precision is still an important and open issue. Strangely enough, even for the solar high-resolution atlas (Kurucz et al. 1984), they concluded that the placement of the continuum is not trivial, and that the influence of many blends gives rise to significant uncertainties.

Our work presents a complete and uniform study of the oxygen abundances in a large sample of stars from the HARPS survey, using two different abundance indicators: the high excitation line at $6158 \AA$ and the oxygen forbidden line at $6300 \AA$. This is the first systematic comparison of these lines in a large sample of main-sequence solar-type stars. The paper is organized as follows: in Sect. 2, we introduce the sample used in this work. The method of the chemical abundance determination, analysis and uncertainties calculations will be explained in Sect. 3. Section 4 is dedicated to the comparison between $6158 \AA$ and $6300 \AA$ as oxygen abundance indicators, where we provide details about their reliability in different ranges of stellar parameters. A discussion of the $[\mathrm{O} / \mathrm{Fe}]$ trends, separation of the different populations of stars and comparison with previous works can be found in Sect. 5. Finally, models of oxygen production in the Galaxy and comparison with observations is presented in Sect. 6 .

\section{Sample description and stellar parameters}

In the past decade a huge effort has been made to discover extrasolar planets. Several radial velocity surveys have carried out a thorough follow-up of solar-type stars over several years, resulting in homogeneous sets of high resolution spectra and large catalogues of uniform stellar parameters (e.g. SWEET-Cat - Santos et al. 2013). Besides the well-known success of such projects for discovering extrasolar planets, these observations give us, for the first time, the opportunity to study the atmospheres and the chemical compositions of solar-type stars with an unprecedented high-quality, homogeneous and extended dataset.

The sample of stars used in this work is a combination of three subsamples from the HARPS planet search programme: HARPS-1 (Mayor et al. 2003), HARPS-2 (Lo Curto et al. 2010) and HARPS-4 (Santos et al. 2011). These stars are mostly slowly rotating chromospherically inactive, unevolved solartype dwarfs and some sub-giants, with spectral types between F2 and M0. The individual spectra were reduced with the HARPS pipeline and then combined using $\mathrm{IRAF}^{1}$ after correcting for its radial velocity. The combined spectra for each star have a resolving power $R \sim 115000$ and a signal-to-noise $(\mathrm{S} / \mathrm{N})$ between $\sim 40$ and $\sim 2000$. About $90 \%$ of the stars have an S/N higher than 100 . This high-quality data allowed us to measure the $6158 \AA$ line in a large sample of stars and investigate its reliability as an oxygen abundance indicator.

We selected 762 stars from the HARPS samples with temperatures higher than $5200 \mathrm{~K}$, for which our stellar parameters are most precise (Tsantaki et al. 2013). Stellar parameters were determined by Sousa et al. (2008, 2011a,b) using the same spectra that we studied. The typical precision uncertainties are of about $30 \mathrm{~K}$ for $T_{\text {eff }}, 0.06 \mathrm{dex}$ for $\log (g), 0.08 \mathrm{~km} \mathrm{~s}^{-1}$ for $\xi_{\mathrm{t}}$, and $0.03 \mathrm{dex}$ for $[\mathrm{Fe} / \mathrm{H}]$. Figure 1 shows the distribution of stellar parameters for our final sample.

1 IRAF is distributed by National Optical Astronomy Observatories, operated by the Association of Universities for Research in Astronomy, Inc., under contract with the National Science Foundation, USA. 
S. Bertran de Lis et al.: Oxygen abundances in G- and F-type stars from HARPS

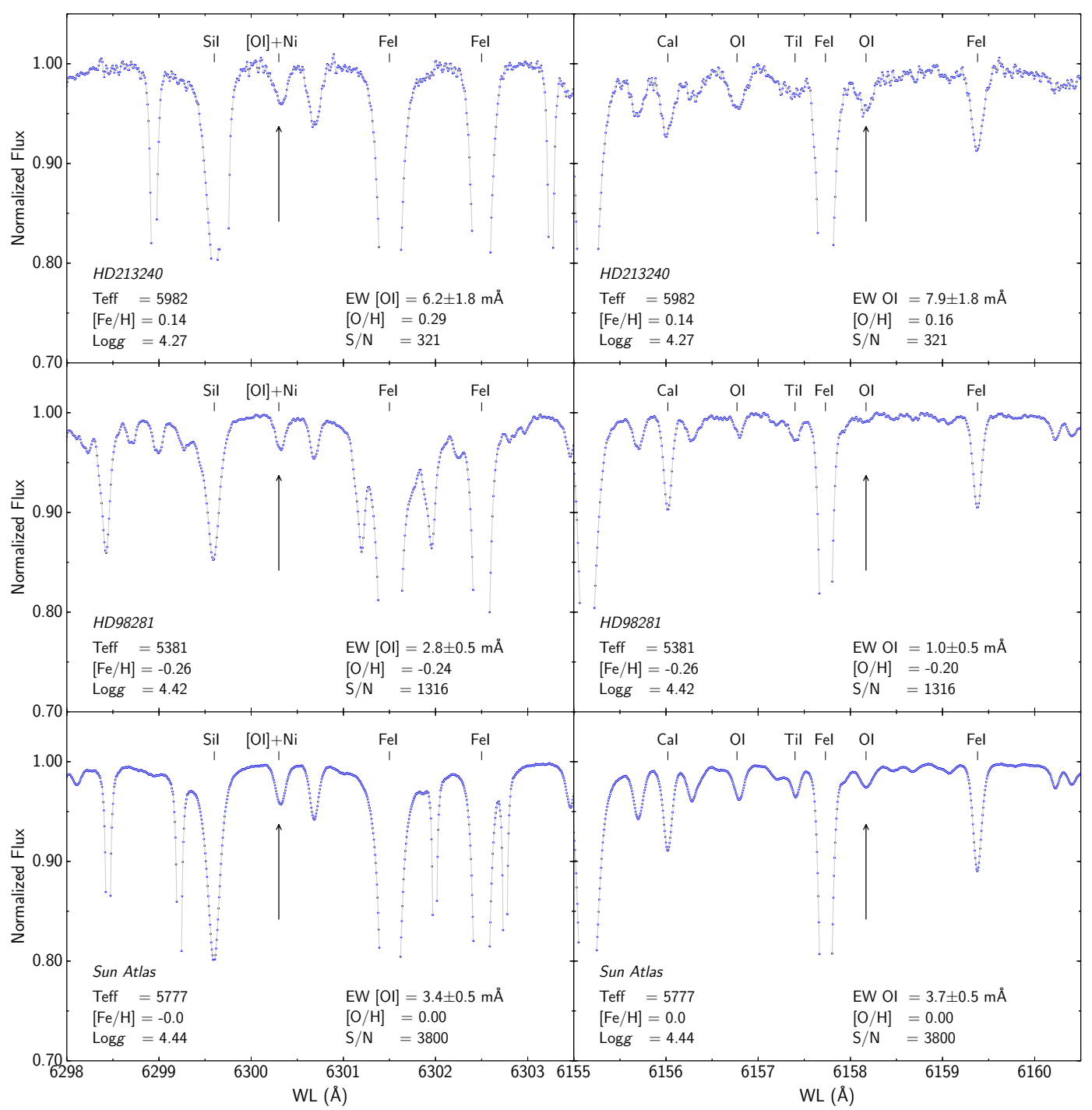

Fig. 2. Spectral regions containing oxygen lines in HD213240, a star from our database with an average $S / N=300$ representative for our catalogue, HD 98281, for which the high S/N allows us to measure lines as small as $1 \mathrm{~m} \AA$, and for the Kurucz Sun Atlas (Kurucz et al. 1984). Stellar parameters, EW of the oxygen lines (Ni blend contribution removed) and LTE abundances are provided in the plot. We note that the provided signal-to-noise ratio is the nominal $\mathrm{S} / \mathrm{N}$.

\section{Abundance analysis}

Oxygen abundances were derived from OI6158 $\AA$ and [OI]6300 ̊ spectral lines. There are two main issues that make the automatic EW measurement of these lines problematic. First, the two lines are placed in complex spectral regions close to some strong FeI lines and, in the case of the $6300 \AA$ transition, also with telluric lines in its vicinity (Fig. 2). Moreover, the strength of these lines in F- and G-type stars is generally smaller than $8 \mathrm{~m} \AA$ (Fig. 3). Altogether, this makes the continuum placement and EW measurement a serious challenge for any automatic code. Therefore, EW measurements were carried out for both lines in a detailed individual analysis using the splot package from IRAF ${ }^{1}$.

In combined spectra the same atmospheric lines can appear at different wavelengths, slightly shifted between them and with different intensities. To account for possible blends with telluric lines, we made a thorough observation of the spectra in the region of the forbidden line. This process was done by identifying the atmospheric pattern, which is repeated along the region of [OI]6300 $\AA$, in a nearby window free from stellar lines. This

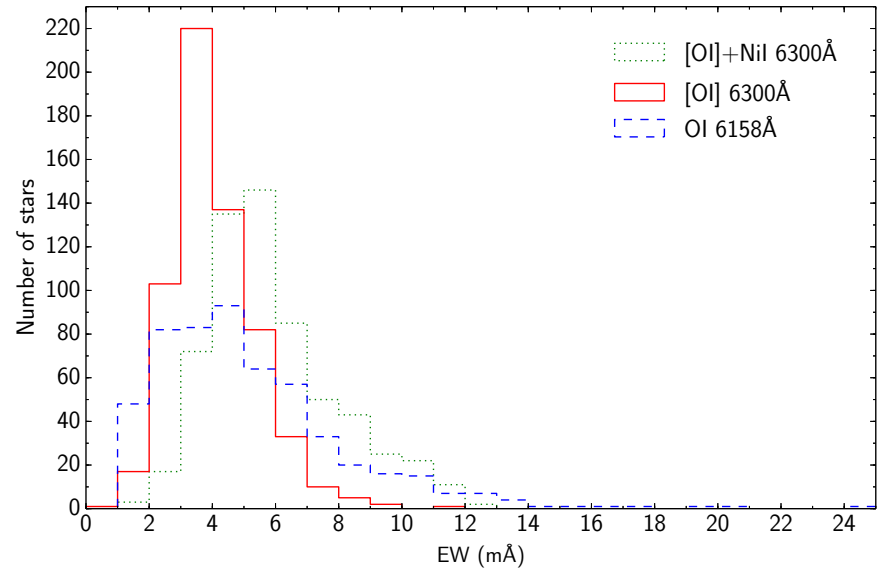

Fig. 3. Distribution of measured EWs for each spectral line.

pattern can be later extrapolated to check if it is superimposed to the $6300 \AA$ line. On the other hand, de-blending was required 

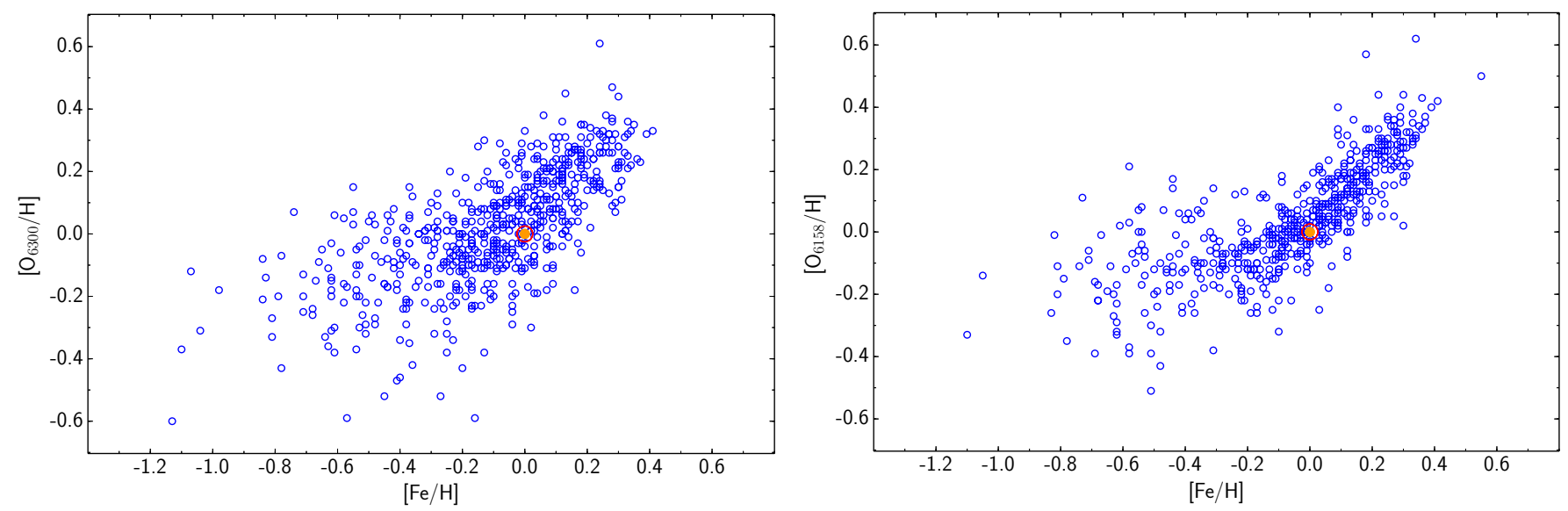

Fig. 4. $[\mathrm{O} / \mathrm{H}]$ vs. $[\mathrm{Fe} / \mathrm{H}]$ for different indicators. The yellow dot corresponds to the solar abundances.

Table 1. Atomic parameters of the spectral lines together with our measurements of EW and abundance in the Sun.

\begin{tabular}{ccccccc}
\hline \hline & $\begin{array}{c}\lambda \\
(\AA)\end{array}$ & $\log (g f)$ & $\begin{array}{c}\chi_{\mathrm{lo}} \\
(\mathrm{eV})\end{array}$ & Ref. & $\begin{array}{c}E W_{\odot} \\
(\mathrm{m} \AA)\end{array}$ & $\log \epsilon(\mathrm{X})_{\odot}$ \\
\hline OI & 6158.171 & -0.296 & 10.74 & 1 & 3.7 & 8.71 \\
{$[\mathrm{OI}]$} & 6300.304 & -9.717 & 0.00 & 1 & 3.4 & 8.65 \\
$\mathrm{NiI}$ & 6300.336 & -2.110 & 4.27 & 2 & 2.0 & 6.25 \\
\hline
\end{tabular}

Notes. This solar value will be used as reference in the present analysis.

References. (1) Caffau et al. (2008); (2) Johansson et al. (2003).

for the $6158 \AA$ feature in order to account for the presence of the FeI line at $6157.5 \AA$. The red wing of this Fe line frequently overlaps with the oxygen line. The spectral windows containing both oxygen spectral lines are shown in Fig. 2. For some still unknown reasons, the nearby line of oxygen at $6156.75 \AA$ provides very different abundances from those based on the $6158 \AA$ and $6300 \AA$ lines. In fact, being even stronger than the line at $6158 \AA$, it has been avoided in previous studies (e.g. Caffau et al. 2008; Takeda \& Honda 2005).

The Solar Atlas of Kurucz (Kurucz et al. 1984) has been used to select continuum regions free from spectral lines. We found that the continuum windows are 6156.94-6157.10 $\AA$ and 6158.80-6158.90 $\AA$ for OI $6158 \AA$, and $6298.20-6298.80 \AA$, $6297.15-6297.45 \AA$ and $6303.00-6303.30 \AA$ for the [OI] $6300 \AA$ line. We have also selected two stars in our sample, with temperatures far from that of the Sun, in order to verify the reliability of those regions at different $T_{\text {eff }}$. This procedure guarantees a uniform continuum placement in our targets, whose spectra are similar to those of the reference stars.

Oxygen abundances from two indicators were determined according to a standard LTE analysis with the revised version of the MOOG2013 spectral synthesis code (Sneden 1973), using the abfind driver and a grid of Kurucz ATLAS9 plane-parallel model atmospheres (Kurucz 1993). Parameters of spectral lines considered in our analysis are listed in Table 1. non-LTE corrections for OI6158 ̊ are negligible (e.g. Caffau et al. 2008) and were not taken into account. On the other hand [OI]6300 $\AA$ is not affected by deviations from LTE. The two oxygen lines have very different excitation energies, but very similar strengths in the Solar Atlas (Kurucz et al. 1984). Once we move towards hotter/cooler stars along the main sequence, one of these lines will become stronger and the other weaker. Thanks to the wide range of $T_{\text {eff }}$ and $[\mathrm{Fe} / \mathrm{H}]$ of the stars in our sample (Fig. 1), we have an interesting opportunity to compare oxygen abundances derived from two lines with very different formation physics. We note that, for still unknown reasons, these lines provide different oxygen abundances in the Sun (Caffau et al. 2008). One of the important questions that will be addressed in this article is whether or not this difference is seen in other sun-like stars. In general, we want to understand which of these lines is more reliable as an oxygen abundance indicator and why.

It is well known that the forbidden line at $6300.30 \AA$ is severely blended with the NiI $6300.34 \AA$ line. The contribution of the NiI line is negligible at $[\mathrm{Fe} / \mathrm{H}]<-0.8$, but becomes very important at solar metallicities (see Sect. 5). The EW of the NiI line in our stars was estimated using the ewfind driver of MOOG and Ni abundances from Adibekyan et al. (2012), calculated from the same spectra and stellar parameters used in this work. The derived EW of the Ni line was subtracted from the total EW of the $6300 \AA$ feature to obtain the contribution of oxygen.

Many authors use the so-called solar $g f$-values to carry out differential abundance analysis, meaning that they force the oscillators strengths of different spectral lines of the same chemical element to provide a unique abundance value. Here we take a different approach. We do not modify the gf-values of the oxygen lines to obtain the same abundance. In this approach we obtain 0.06 dex difference between the solar oxygen derived from the $6158 \AA$ and $6300 \AA$ lines. We want to investigate whether this difference exists in other sun-like stars with a quality of spectra similar to the Solar Atlas. The solar abundances listed in Table 1 are used as a reference in the analysis.

Abundances from OI6158 $\AA$ were obtained for 535 stars, while 610 stars were analysed using [OI]6300 A from the initial sample of 762 stars. The final results from both lines are shown in Fig. 4. These are presented relative to the solar reference abundance (Table 1), derived following the same procedure as for the rest of the sample. Tables 2 and 3 provide the abundances from the OI6158 $\AA$ and [OI]6300 $̊$ lines, respectively, together with the stellar parameters, the EW of the lines and its total uncertainty.

In Fig. 5 we plot oxygen abundances against stellar parameters. Such plots reveal systematic effects that could influence our measurements. We note a slight dependence of $\left[\mathrm{O}_{6300} / \mathrm{H}\right]$ on $T_{\text {eff }}$ at temperatures less than $5600 \mathrm{~K}$, for which lower $[\mathrm{O} / \mathrm{H}]$ abundances are found. Although restricted to the low temperature end, a trend with opposite sign can also be noticeable for $\left[\mathrm{O}_{6158} / \mathrm{H}\right]$. Nevertheless, if we only consider stars with 

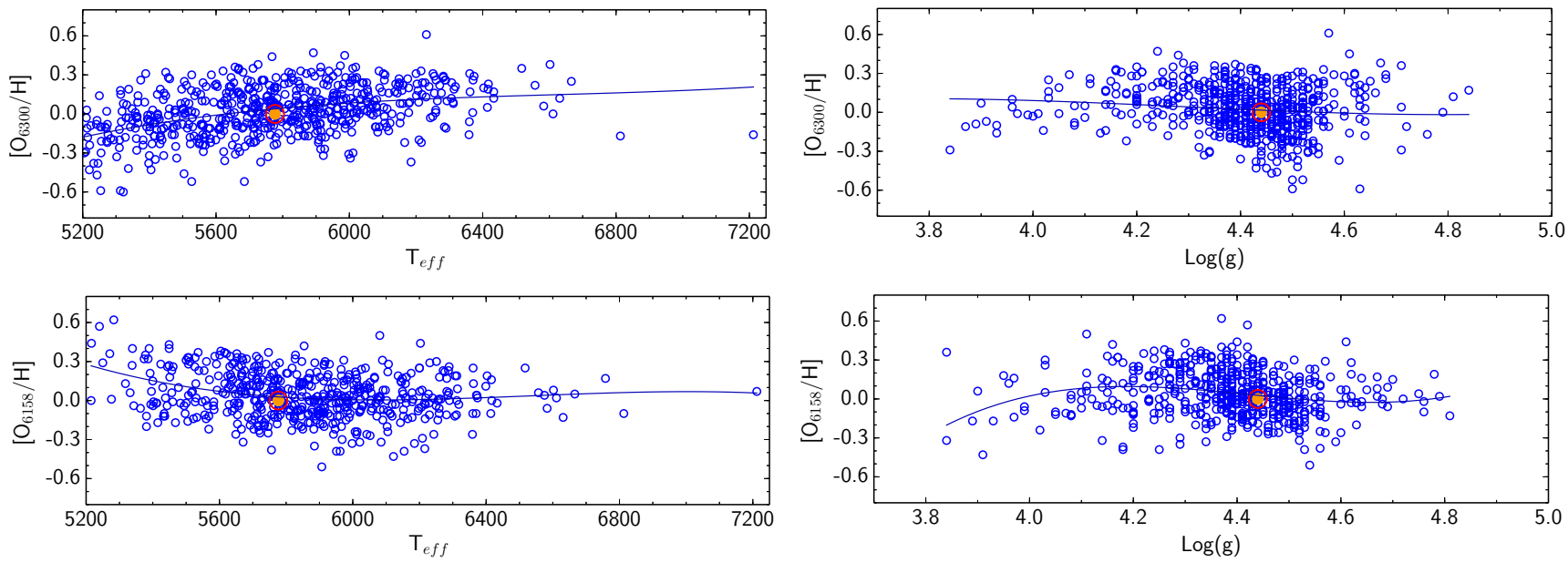

Fig. 5. Systematic trends of $[\mathrm{O} / \mathrm{H}]$ for different indicators with temperature and surface gravity. The solid line is the cubic best fit.

Table 2. Oxygen abundances from the OI 6158 Å line.

\begin{tabular}{lcccccccc}
\hline \hline Star & $\begin{array}{c}T_{\text {eff }} \\
(\mathrm{K})\end{array}$ & $\begin{array}{c}\log (g) \\
\left(\mathrm{cm} \mathrm{s}^{-2}\right)\end{array}$ & $\begin{array}{c}\xi_{\mathrm{t}} \\
\left(\mathrm{km} \mathrm{s}^{-2}\right)\end{array}$ & {$[\mathrm{Fe} / \mathrm{H}]$} & $\begin{array}{c}E W_{\text {OI }} \\
(\mathrm{m} \AA)\end{array}$ & $\begin{array}{c}\delta E W_{\text {OI }} \\
(\mathrm{m} \AA)\end{array}$ & {$[\mathrm{O} / \mathrm{H}]_{\mathrm{OI}}$} & Gal. Pop. \\
\hline Sun & 5777 & 4.44 & 1.00 & 0.00 & 3.7 & 0.3 & 0.00 & thin \\
HD 70889 & 6051 & 4.49 & 1.13 & 0.11 & 6.9 & 0.5 & 0.10 & thin \\
HD 21161 & 5923 & 4.24 & 1.14 & 0.09 & 7.5 & 2.8 & 0.18 & thin \\
HD 37226 & 6178 & 4.16 & 1.61 & -0.12 & 7.4 & 0.8 & -0.01 & thin \\
HD 125881 & 6036 & 4.49 & 1.10 & 0.06 & 5.8 & 0.6 & 0.04 & thin \\
HD 117105 & 5889 & 4.41 & 1.13 & -0.29 & 3.0 & 0.7 & -0.12 & thin \\
$\ldots$ & $\ldots$ & $\ldots$ & $\ldots$ & $\ldots$ & $\ldots$ & $\ldots$ & $\ldots$ & $\ldots$ \\
\hline
\end{tabular}

Notes. The full table is available at the CDS.

Table 3. Oxygen abundances from the [OI] $6300 \AA$ A line.

\begin{tabular}{lcccccccccc}
\hline \hline Star & $\begin{array}{c}T_{\text {eff }} \\
(\mathrm{K})\end{array}$ & $\begin{array}{c}\log (g) \\
\left(\mathrm{cm} \mathrm{s}^{-2}\right)\end{array}$ & $\begin{array}{c}\xi_{\mathrm{t}} \\
\left(\mathrm{km} \mathrm{s}^{-2}\right)\end{array}$ & {$[\mathrm{Fe} / \mathrm{H}]$} & {$[\mathrm{Ni} / \mathrm{H}]^{a}$} & $\begin{array}{c}E W_{\mathrm{Ni}} \\
(\mathrm{m} \AA)\end{array}$ & $\begin{array}{c}E W_{[\mathrm{OI}]} \\
(\mathrm{m} \AA)\end{array}$ & $\begin{array}{c}\delta E W_{\text {[OI] }} \\
(\mathrm{m} \AA)\end{array}$ & {$[\mathrm{O} / \mathrm{H}]_{[\mathrm{OI}]}$} & Gal. Pop. \\
\hline Sun & 5777 & 4.44 & 1.00 & 0.00 & 0.00 & 2.0 & 3.4 & 0.3 & 0.00 & thin \\
HD 48611 & 5337 & 4.51 & 0.69 & -0.36 & -0.37 & 1.2 & 1.9 & 0.8 & -0.42 & thin \\
HD 70889 & 6051 & 4.49 & 1.13 & 0.11 & 0.09 & 1.8 & 3.3 & 0.4 & 0.10 & thin \\
HD 21161 & 5923 & 4.24 & 1.14 & 0.09 & 0.11 & 2.2 & 5.9 & 2.9 & 0.23 & thin \\
HD 130322 & 5365 & 4.37 & 0.90 & -0.02 & -0.03 & 2.5 & 5.1 & 1.0 & 0.09 & thin \\
HD 222422 & 5475 & 4.46 & 0.73 & -0.12 & -0.15 & 1.8 & 2.8 & 0.8 & -0.16 & thin \\
$\ldots$ & $\ldots$ & $\ldots$ & $\ldots$ & $\ldots$ & $\ldots$ & $\ldots$ & $\ldots$ & $\ldots$ & $\ldots$ & $\ldots$ \\
\hline
\end{tabular}

Notes. ${ }^{(a)}[\mathrm{Ni} / \mathrm{H}]$ from Adibekyan et al. (2012). The full table is available at the CDS.

temperatures larger than $\sim 5400 \mathrm{~K}$ (e.g. when the $6158 \AA$ line is strong enough for reliable measurements) we will find no temperature dependence for the $6158 \AA$ line. In general, given the high abundance scatter observed in these graphs, the small dependence on $T_{\text {eff }}$ for cool stars can be neglected. No gravity effect was found.

\subsection{Uncertainties}

There are several sources of random error involved in the process of measuring chemical abundances. These are related either to the measurement of the EW or to the calculation of the abundances. It is not easy to define the contribution of each error and account analytically for them. In the present work we have considered three different contributions to the uncertainties in our measurements.
First, we studied the sensitivity of the abundances to the stellar parameters: effective temperature $T_{\text {eff }}$, metallicity $[\mathrm{Fe} / \mathrm{H}]$, surface gravity $\log (g)$ and microturbulence $\xi_{\mathrm{t}}$. Variations of these parameters introduce small changes in the abundances which, regardless of the parameter we vary, also depend on the $T_{\text {eff }}$ of the star (Adibekyan et al. 2012). Therefore, we will carry on our analysis with three different sub-samples based on the $T_{\text {eff }}$ : $T<5377,5377 \leq T \leq 6177$ and $T>6177$. The middle group comprises the stars with $T_{\text {eff }}=T_{\odot} \pm 400 \mathrm{~K}$. We expect that the sensitivity to the stellar parameters behave similar within each range of temperature. The average values of the stellar parameters for each group are shown in Table 4. These parameters together with the average oxygen abundance on each group are used to compute theoretical EWs of oxygen lines using the ewfind driver of MOOG. Keeping the EW constant, we then calculate abundances by varying, one by one, the stellar parameters by an amount equal to their uncertainty (Table 5). Among all 
Table 4. Average stellar parameters for the three sub-samples of different $T_{\text {eff }}$.

\begin{tabular}{ccccc}
\hline \hline & $\begin{array}{c}T_{\text {eff }} \\
(\mathrm{K})\end{array}$ & $\log (g)$ & {$[\mathrm{Fe} / \mathrm{H}]$} & $\begin{array}{c}\xi_{\mathrm{t}} \\
\left(\mathrm{km} \mathrm{s}^{-1}\right)\end{array}$ \\
\hline Low $T_{\text {eff }}$ & 5292 & 4.35 & -0.16 & 0.73 \\
Solar $T_{\text {eff }}$ & 5774 & 4.40 & -0.12 & 1.00 \\
High $T_{\text {eff }}$ & 6340 & 4.50 & -0.10 & 1.59 \\
\hline
\end{tabular}

the values, we select as the final error the largest deviation from the original oxygen value. $6158 \AA$ appears to be more sensitive to $T_{\text {eff }}$ while $6300 \AA$ suffers from larger variations with $\log (g)$.

Another source of uncertainty is the statistical photometric error due to the noise in each pixel. We can evaluate the partial derivative of the EW with respect to the flux (Bohlin et al. 1983; Cayrel 1988) to obtain an analytical solution to this contribution:

$$
E W=\sum\left(\Delta \lambda \frac{C_{i}-I_{i}}{C_{i}}\right), \delta E W=\sqrt{\sum_{i=1}^{N}\left(\frac{\partial E W}{\partial I_{i}}\right)^{2}}=2.45 \epsilon \sqrt{\sigma \Delta \lambda} .
$$

Where $C_{i}$ is the continuum flux level, $I_{i}$ is the flux of each measurement, $\Delta \lambda$ represents the wavelength increment per pixel ( $0.01 \AA$ for HARPS spectra), $\epsilon$ is the rms relative photometric accuracy in the continuum and $\sigma$ is the standard width over which we sum six times assuming a Gaussian profile. We determine the standard width theoretically as the quadratic sum of different broadenings: instrumental (wavelength divided by the spectral resolution of the instrument), macroturbulence based on the relation provided by Valenti \& Fischer (2005), microturbulence, rotation (using an average value of $3 \mathrm{~km} \mathrm{~s}^{-1}$ ) and thermal broadening. The latter is calculated with (Gray 1992):

$$
\Delta \lambda_{D}=\frac{\lambda}{c} \sqrt{\frac{2 k T}{m}}=4.301 \times 10^{-7} \lambda \sqrt{\frac{T}{\mu}} .
$$

Where $k$ is Boltzmann's constant, T represents the effective temperature and $\mu$ is the atomic weight in atomic mass units.

The last and the greatest contribution to the uncertainties is related to the measurement of the EW and its sensitivity to the continuum placement. Although this error can reach a large percentage of the EW, it is commonly ignored by many authors, resulting in significantly underestimated uncertainties in the abundances. In order to quantify this contribution, one should calculate the difference between the areas of the Gaussian fits by placing the continuum in different positions. Given the high $\mathrm{S} / \mathrm{N}$ of our spectra, an therefore the low uncertainty in the rms, the possible continuum placements will yield small variations between the respective Gaussian depths. Thus, the error in EW can be approximated as the area of the rectangle between the best and the $\pm \epsilon$ continuum placements, where $\epsilon$ is the inverse of the $\mathrm{S} / \mathrm{N}$ per resolution element. The width at the continuum level is again equal to $6 \sigma$. All together yields an error of $\pm 6 \sigma \epsilon$.

Figure 6 shows the significance of different contributions to the total uncertainty of the EW, which is similar for both oxygen lines. We have converted the error in abundance due to uncertainties in stellar parameters to the corresponding EW error using the ewfind driver of MOOG synthesis code. Each contribution to the EW is finally added quadratically to get the one- $\sigma$ uncertainty. Because of the non-linear dependence of the abundance on the EW, asymmetric errors will arise when the EW error is propagated to get abundance uncertainties. For further details on the determination of the abundance uncertainty we refer the reader to Appendix A.

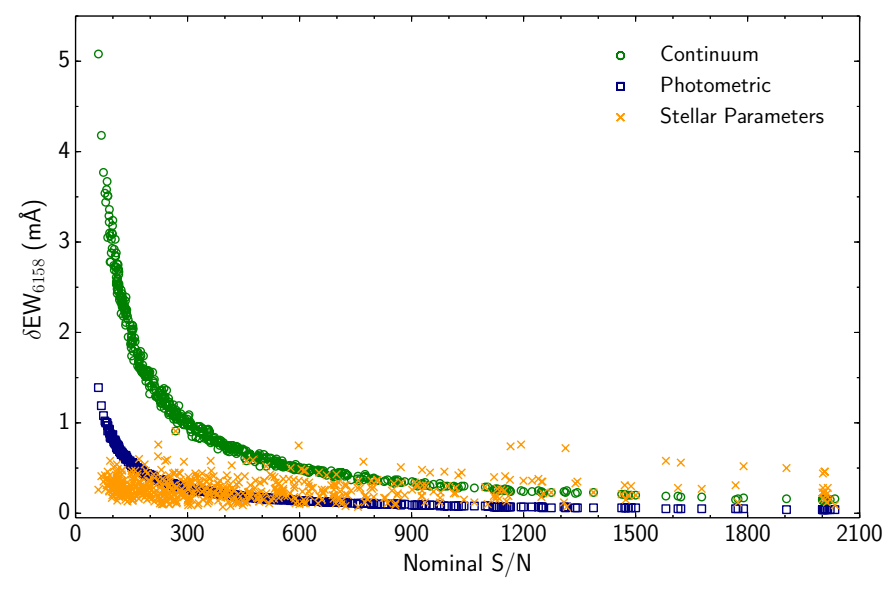

Fig. 6. Contribution to the total EW error of OI6158 $\AA$ of each source of uncertainty.

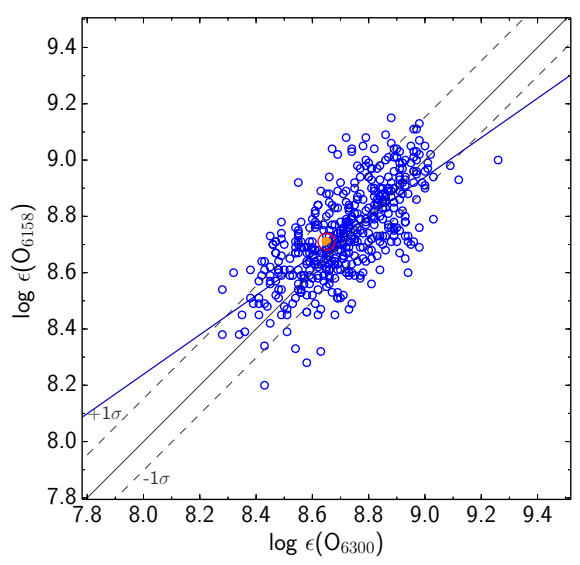

Fig. 7. Comparison of the absolute oxygen abundances from OI6158 $\AA$ with those obtained from [OI]6300 $\AA$ lines. Solid lines represent the diagonal and the linear fit to the data. Dashed lines enclose $68 \%$ of the data around the mean $(0.024 \mathrm{dex})$.

\section{Comparison between indicators}

Abundances from both the OI6158 $\AA$ and [OI]6300 $\AA$ lines were measured in 447 stars from the initial sample of 762 . This large number of stars covers a wide range of temperatures and metallicities, which allows us to carry out a comprehensive comparison of the performance of these spectral lines as oxygen abundance indicators. In this section we study the agreement between chemical abundances obtained from both spectral lines, and we also investigate their reliability and usability related to the stellar parameters.

In Sect. 3 we have already noted that the absolute oxygen abundances from the two lines in the Sun disagree, since $\log \epsilon\left(\mathrm{O}_{6158}\right)$ is 0.06 dex higher than $\log \epsilon\left(\mathrm{O}_{6300}\right)$. The comparison for all the stars with common measurements is shown in Fig. 7. The OI6158 A line provides higher abundances in stars with $[\mathrm{O} / \mathrm{H}]$ similar or lower than the Sun, while $[\mathrm{OI}] 6300 \AA$ yields higher values for oxygen-rich stars. This small trend could be due to overestimation of the EW when the lines are small. However, the trend is not significant as it falls within the standard deviation of $\pm 1 \sigma=0.127$ dex. On average, absolute oxygen abundances derived from the $6158 \AA$ spectral line are 0.024 dex higher than [OI]6300 $\AA$. This value is considerably smaller than the typical uncertainties of the oxygen abundances. 
Table 5. Abundance sensitivities to the stellar parameters of both oxygen indicators for the three sub-samples of different $T_{\text {eff }}$.

\begin{tabular}{llcrrrrrrr}
\hline \hline & & \multicolumn{2}{c}{$\Delta T_{\text {eff }}$} & \multicolumn{2}{c}{$\Delta \log (g)$} & \multicolumn{2}{c}{$\Delta[\mathrm{Fe} / \mathrm{H}]$} & \multicolumn{2}{c}{$\Delta \xi_{\mathrm{t}}$} \\
& & $+30 \mathrm{~K}$ & $-30 \mathrm{~K}$ & $+0.06 \mathrm{dex}$ & $-0.06 \mathrm{dex}$ & $+0.03 \mathrm{dex}$ & $-0.03 \mathrm{dex}$ & $+0.08 \mathrm{~km} \mathrm{~s}^{-1}-0.08 \mathrm{~km} \mathrm{~s}^{-1}$ \\
\hline \multirow{2}{*}{ Low $T_{\text {eff }}$} & $\Delta$ OI 6158 & -0.03 & +0.04 & +0.02 & -0.01 & 0.00 & +0.01 & 0.00 & +0.01 \\
& $\Delta[$ OI]6300 & +0.01 & 0.00 & +0.03 & -0.02 & +0.02 & -0.01 & 0.00 & 0.00 \\
\multirow{2}{*}{ Solar $T_{\text {eff }}$} & $\Delta$ OI 6158 & -0.02 & +0.03 & +0.02 & -0.01 & 0.00 & +0.01 & 0.00 & 0.00 \\
& $\Delta$ [OI]6300 & +0.01 & 0.00 & +0.03 & -0.02 & +0.01 & -0.01 & 0.00 & 0.00 \\
\multirow{2}{*}{ High $T_{\text {eff }}$} & $\Delta$ OI 6158 & -0.02 & +0.02 & +0.02 & -0.02 & 0.00 & +0.01 & 0.00 & 0.00 \\
& $\Delta$ [OI]6300 & +0.01 & -0.01 & +0.02 & -0.02 & +0.01 & -0.01 & 0.00 & 0.00 \\
\hline
\end{tabular}

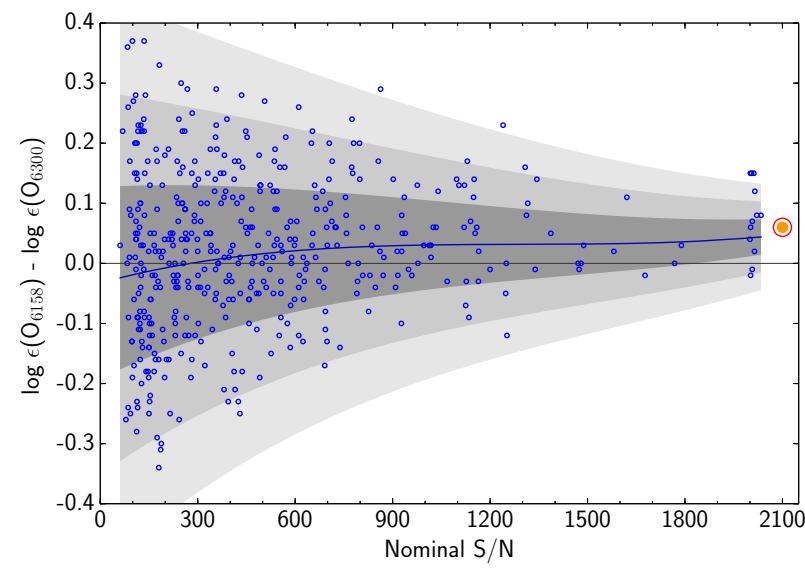

Fig. 8. Difference between absolute oxygen abundances derived from the OI6158 $\AA$ and $[\mathrm{OI}] 6300 \AA$ lines against nominal signal-to-noise ratio. Weighted moving average (boxcar average) of data points is shown as a solid line, together with the 1,2 , and 3 sigma dispersion regions in different grey shades. The Sun has been shifted towards lower $\mathrm{S} / \mathrm{N}$ for plotting purposes.

No conclusion can be drawn, except that on average both spectral lines yield compatible values of oxygen abundance within the uncertainties.

As we move towards stars with better quality spectra, i.e. higher $\mathrm{S} / \mathrm{Ns}$, the abundance uncertainties decrease. As expected, our measurements become more precise and we achieve a smaller dispersion in the difference between oxygen indicators. Specifically, while the standard deviation for the whole sample was $0.127 \mathrm{dex}$, at high $\mathrm{S} / \mathrm{N}$ it drops to 0.072 dex. However, for stars with $S / N>1000$ the average difference in oxygen abundances is $0.051 \mathrm{dex}$, which is compatible with previously measured differences in the Sun (Caffau et al. 2008). These deviations increase as we consider higher S/Ns, indicating a possible discordance between both indicators. The agreement between abundance indicators as a function of the $\mathrm{S} / \mathrm{N}$ is presented in Fig. 8. We have performed a weighted moving average (500 steps, step size 50 and weigthed with $1 / \sigma^{2}$ ) that has been smoothed by a factor of 0.4 . The 1,2 and 3 standard deviations levels from the mean, which are represented by shaded areas in the figure, clearly show how the dispersion decreases at better $\mathrm{S} / \mathrm{N}$, while the mean is shifted towards higher values of $06158 \AA$. This result may suggest that the 0.06 dex disagreement found in the Sun is not unique, and may have some physical explanation.

The different sensitivities of the two oxygen lines to stellar parameters allows us to define windows where each of these lines can be used more or less easily. The EW of both spectral lines is smaller than $8 \mathrm{~m} \AA$ at $T_{\text {eff }}>5800 \mathrm{~K}$. However, because of

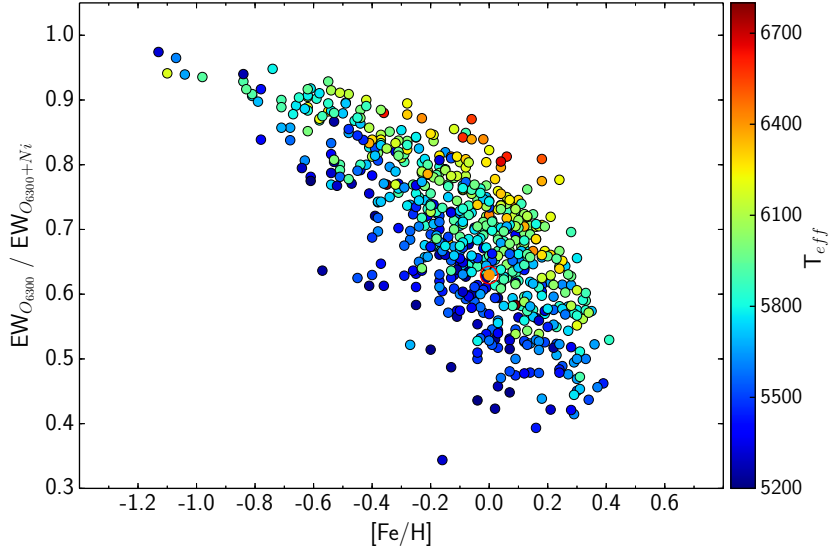

Fig. 9. Fraction of oxygen in the EW of the $6300 \AA$ feature as a function of metallicity. Dependence on the effective temperature is shown in colours.

the high excitation energy of $6158 \AA$, this line becomes significantly stronger in hotter stars. Specifically, the EW of OI6158 is always larger than the EW of [OI]6300 $\AA$ in all the stars with $T_{\text {eff }}>6200 \mathrm{~K}$.

It is also important to note the uncertainties that affect each line. Because of the density of spectral features in the range of $6158 \AA$, the largest source of error in the EW measurement is the continuum placement. Therefore, it is more complicated to measure this line in cool stars, where in addition to many blends, the line itself is very weak. The $6300 \AA$ feature can be considered as problematic in solar-type stars for two important reasons. The first is related to the presence of numerous telluric lines often severely blended with the oxygen feature. Even more serious is the problem with the $\mathrm{Ni}$ absorption line (see above). Johansson et al. (2003) provided a $\log (g f)$ value with $14 \%$ uncertainty. Our calculations show that the Ni line can account for up to $60 \%$ of the EW of the $6300 \AA$ absorption feature in cool, metal-rich stars (see Fig. 9). Thus, the abundance of $\mathrm{Ni}$ and its atomic parameters plays a key role in this topic. As an illustration, the error in the Ni $\log (g f)$ is introducing an uncertainty in the oxygen EW measurement of HD 142 equal to $3.1_{-0.1}^{+0.2} \mathrm{~m} \AA$, which is translated into $[\mathrm{O} / \mathrm{H}]=0.2_{-0.00}^{+0.03}$ dex. In stars with medium to low $\mathrm{S} / \mathrm{N}$ this contribution will be smaller than the two main sources of uncertainty considered in this work. Even though, the larger scatter found in Fig. 4 with $6300 \AA$ could be partially explained by the uncertainty in the $\mathrm{Ni} \log (g f)$, in combination with the possible presence of undetected telluric lines slightly above the level of the noise.

The main and the most important aspect of the $6158 \AA$ line is that it does not suffer from known blends. It is as strong as 


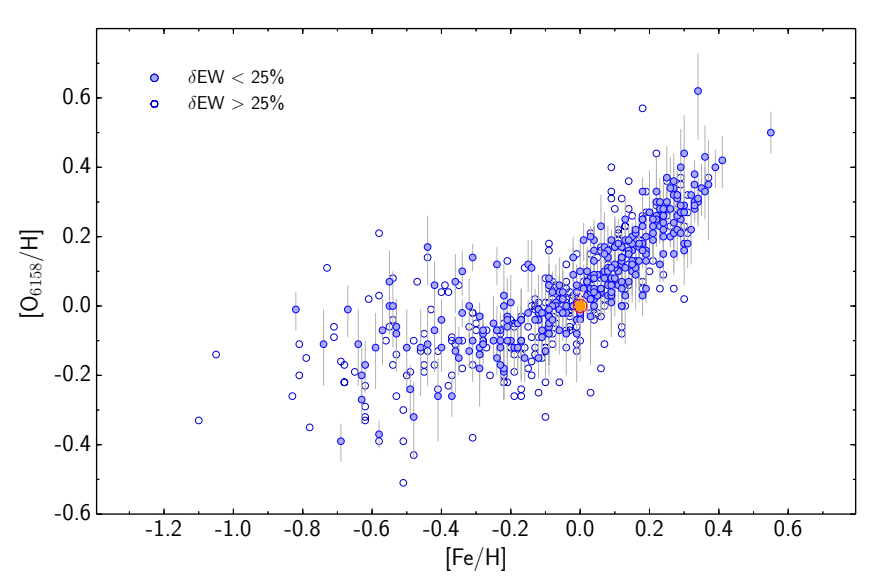

Fig. 10. $[\mathrm{O} / \mathrm{H}]$ vs. $[\mathrm{Fe} / \mathrm{H}]$ for $\mathrm{OI} 6158 \AA$ A. Filled (open) circles represent data for which EW measurements have an uncertainty under (above) 25\%. Absolute error bars are over-plotted for our best measurements.

the forbidden line in solar analogues and stronger than $6300 \AA$ at temperatures higher than $6200 \mathrm{~K}$. Our results suggest that the $6158 \AA$ line can be used as a reliable indicator of oxygen abundance in solar-type stars. However, we should bear in mind that it may suffer non-negligible non-LTE effects in hot stars (Sitnova et al. 2013).

\section{Oxygen trends in the thin and thick disks}

\subsection{Observed dispersion of $[\mathrm{O} / \mathrm{H}]$}

The abundance scatter seen in the Galactic trends of many chemical elements is very small. Adibekyan et al. (2012) carried out a homogeneous study and found small abundance dispersion in such elements as $\mathrm{Si}$ or Ti. The abundances presented here have been derived from the same spectra and stellar parameters. Thus, using the same database and techniques as Adibekyan et al. (2012) we would expect to get equally precise and trustworthy results for other elements. However, those elements have many more reliable spectral lines for abundance analysis, and therefore is not surprising that we found a wider scatter in the case of oxygen (Fig. 4). Even so, scatter found in the present work for $\left[\mathrm{O}_{6158} / \mathrm{H}\right]$ is comparable to that of $\mathrm{Mg}$, which was derived using three spectral lines (Adibekyan et al. 2012). Petigura \& Marcy (2011) suggested an astrophysical origin to explain the dispersion found in oxygen trends.

We consider a sub-sample of 296 stars, $55 \%$ of the sample, for which we have errors smaller than $25 \%$ in EW in order to verify whether the observed abundance scatter of oxygen is due to the lack of precise measurements. We also restricted the analysis to the OI 6158 line, since we found in this study that it is more trustworthy. We see in Fig. 10 that the scatter is still very high in this "most precise" sub-sample at $[\mathrm{Fe} / \mathrm{H}]<-0.2$ dex. On the other hand, at higher metallicities the scatter is not remarkably reduced by selecting the best measurements, and only outliers are removed. Error bars are about the size of the dispersion and it is therefore difficult to draw any conclusion about the origin of the scatter.

The observed scatter at a given metallicity is expected to have two contributions: a real star-to-star scatter, and also a dispersion due to the uncertainties in our measurements. We have performed a moving average ( 250 steps with 0.03 dex width) to derive both the average $[\mathrm{O} / \mathrm{H}]$ with their respective scatter, and

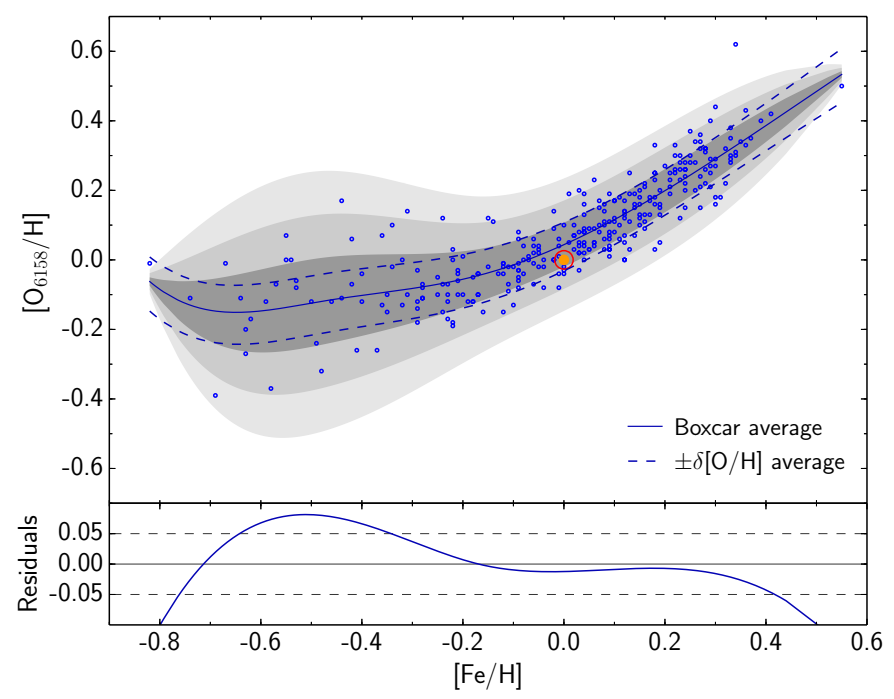

Fig. 11. Upper panel: $[\mathrm{O} / \mathrm{H}]$ vs. $[\mathrm{Fe} / \mathrm{H}]$ for OI $6158 \AA$. Only stars with EW relative error below $25 \%$ are considered. Solid line represent the average oxygen abundance. The 1, 2 and 3 sigma levels of dispersion around the mean are presented as shaded areas. Dashed line shows the average uncertainties. For plotting purposes the average uncertainties are shown around the mean abundance. Lower panel: difference between the $\pm 1 \sigma$ width of the scatter around the mean and the $\pm 1 \sigma$ width of the uncertainties.

the mean $\pm \delta[\mathrm{O} / \mathrm{H}]$ as a function of metallicity. The goal of this test is to find out which of the two contributions to the scatter dominates our sample. Results of the test are depicted in Fig. 11. We are aware that this test is not valid at the edges of the metallicity range due to the undersampling of the bins, which makes unreliable the provided mean values.

In order to evaluate the relative importance of the contributions to the scatter, in the lower panel of the figure we show the difference between the widths of the $\pm 1 \sigma$ regions. A negative residual would imply an overestimation of our uncertainties, while a positive residual could be due to either a real scatter or to an underestimation of the errors. Since the uncertainties have been derived with the same method for all stars, and there is no dependency on metallicity, differences in residuals as a function of metallicity are expected to be caused by real star-to-star dispersion. At high metallicities the observed scatter and the uncertainties are comparable, and do not allow us to detect the possible presence of an astrophysical scatter. However, the positive residual found at $[\mathrm{Fe} / \mathrm{H}]<-0.2$ dex reveals a real star-to-star scatter that cannot be explained by uncertainties. The results of this test are not surprising, and they are in perfect agreement with the fact that at $[\mathrm{Fe} / \mathrm{H}]<-0.2$ dex there is a mixture of stars from two different populations of the Galactic disk (see below for details). However, this expected results confirm the effectiveness of this method to show up the presence of a real scatter. In addition, the satisfactory agreement between uncertainties and scatter at high metallicities support the validity of our uncertainty calculations. Given that the test was performed using a sub-sample of the best measurements, this last result should consequently be interpreted with caution. A similar test with the whole sample would show a slight overestimation of the uncertainties (negative residuals), due to the exponential growth of the errors as we move towards lower $\mathrm{S} / \mathrm{N}$. Therefore, we conclude that although our uncertainty calculations are overestimated at very low $\mathrm{S} / \mathrm{N}$, they appear to satisfactory account for the observed scatter at medium to high $\mathrm{S} / \mathrm{N}$. 

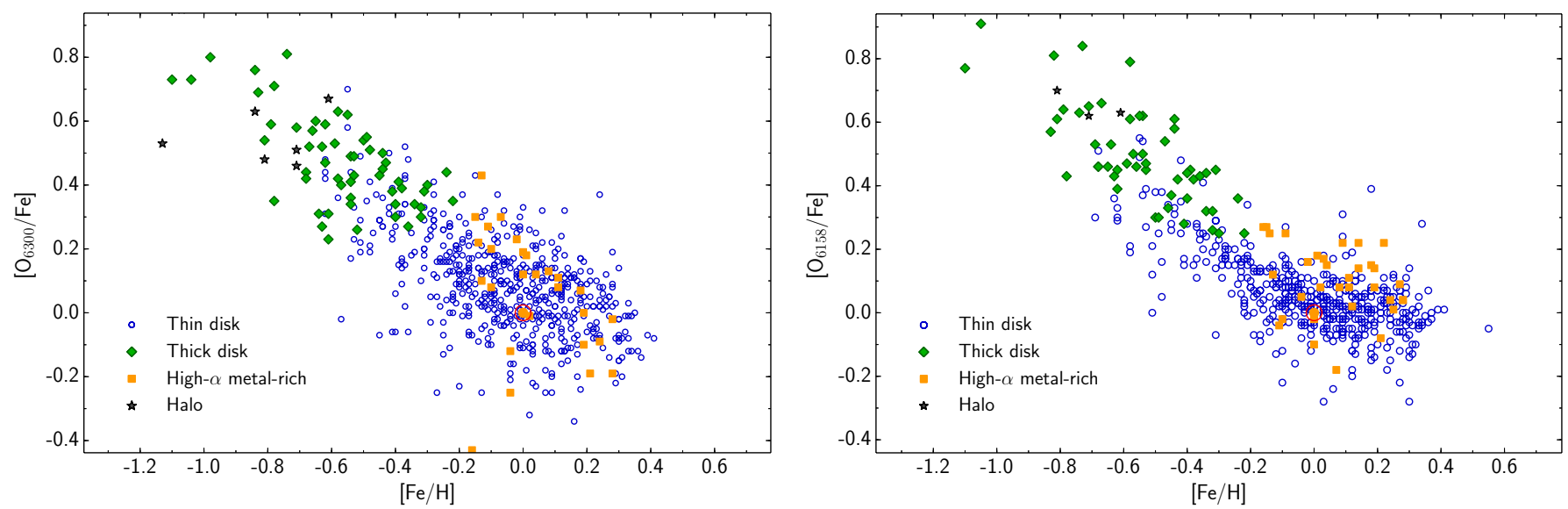

Fig. 12. $[\mathrm{O} / \mathrm{Fe}]$ vs. $[\mathrm{Fe} / \mathrm{H}]$ for $[\mathrm{OI}] 6300 \AA$ and $\mathrm{OI} 6158 \AA$. Stars from different populations are separated according to their chemical composition.

\subsection{Thin and thick disk separation}

It is believed that $\alpha$ elements, such as oxygen, are produced in massive stars that enrich the interstellar medium when they explode as type II supernova. By contrast, iron peak elements are mostly synthesized in SNe Ia explosions (e.g. Thielemann et al. 2002). The oxygen over iron ratio can provide hints on the formation of the Galactic disk and its chemical history, but the observed Galactic trends are diffused as a result of the mixture of stars from two different populations in the studied metallicity regime.

The chemical distinction between thin and thick disks was first noticed by Fuhrmann (1998) and further investigated by Prochaska et al. (2000), among others. We have classified the stars in our sample between thin disk, high- $\alpha$ metal-poor (thick disk) and high- $\alpha$ metal-rich stars according to chemical criteria described in Adibekyan et al. (2011). These authors show that disk populations are clearly separated in terms of $[\alpha / \mathrm{Fe}]$, where " $\alpha$ " refers to the average abundance of $\mathrm{Mg}, \mathrm{Si}$, and Ti. The ratio of oxygen over iron for the different disk populations is presented in Fig. 12. There is no obvious difference seen between the thick and the thin populations from [OI]6300, which can be attributed to the larger errors and scatter. We see a clear overabundance of oxygen in thick disk stars once the OI6158 line is considered. Moreover, using the sub-sample with the most precise abundance values (Fig. 13), we can check that none of the thick disk stars falls below the trend for the thin disk, although few thin disk stars have $[\mathrm{O} / \mathrm{Fe}]$ higher than expected for this population. In addition, both trends appear to be remarkably separated at lower metallicities. Thus, we can confirm with our data that oxygen, just like the other $\alpha$ elements, is enhanced in the thick disk stars.

The high- $\alpha$ metal-rich population (h $\alpha \mathrm{mr}$ ) was presented by Adibekyan et al. $(2011,2013)$ as a new population due to the gap in metallicity found between the latter and the thick disk. In addition, a gap in the distribution of $[\alpha / \mathrm{Fe}]$ at 0.17 was also noticed. The present work is based on the same sample as Adibekyan et al. (2011), and therefore our data also show a gap in metallicity. The separation in the $[\mathrm{O} / \mathrm{Fe}]$ distribution is not as clear as in $[\alpha / \mathrm{Fe}]$ because both populations are mixed around $[\mathrm{O} / \mathrm{Fe}] \sim 0.25$, probably caused by the larger errors in the determination of oxygen abundances compared to other $\alpha$ elements. Our most precise sample show that all the stars from the h $\alpha \mathrm{mr}$ population fall above the average trend for thin disk (Fig. 13), and thus we can confirm that homr stars are enhanced in $[\alpha / \mathrm{Fe}]$

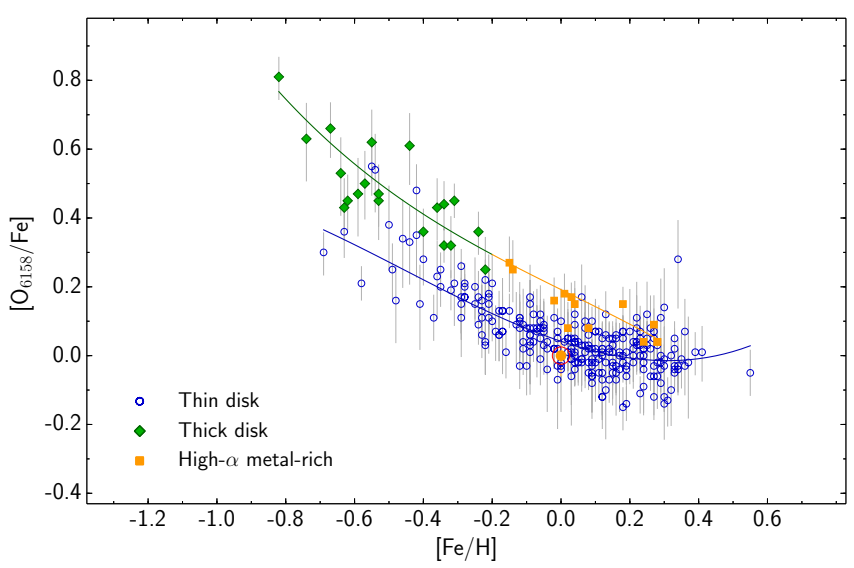

Fig. 13. $[\mathrm{O} / \mathrm{Fe}]$ vs. $[\mathrm{Fe} / \mathrm{H}]$ for the stars with $\delta E W<25 \%$. Stars from different populations are separated according to their chemical composition. Solid lines represent the fitted trend, weighted with the uncertainties, for each population (thick and homr stars are fitted as a single population). Absolute error bars are over-plotted.

(where $\alpha$ refers here to oxygen). Recent studies by Bensby et al. (2014) and Helmi et al. (2014) also confirmed the alpha enhancement of a metal-rich population, nevertheless, they did not observe a gap in metallicity between them and the thick disk stars. Bensby et al. (2014) argued that the high- $\alpha$ metal-rich family might be a metal rich tail of the thick disk. With this new oxygen abundance determination we cannot draw conclusions about this population, i.e. whether is distinct or just a continuation of the thick disk towards higher metallicities.

Once the disk populations are separated, we can revisit the issue about the origin of the scatter in the observed $[\mathrm{O} / \mathrm{H}]$ trends. We repeat the test performed for the best sample, but this time independently for the thin and thick (including h $\alpha \mathrm{mr}$ ) disk populations. The moving average for the oxygen abundances and $\pm \delta[\mathrm{O} / \mathrm{H}]$ uncertainties are shown in Fig. 14, together with the residuals of the $\pm 1 \sigma$ width regions. Given the small residuals found between uncertainties and scatter for thick and homr population, and taking into account the reduced number of stars that we have in this group, no conclusion can be drawn about the existence of a real star-to-star dispersion. In contrast, thin disk stars seem to show a positive residual at $-0.2<[\mathrm{Fe} / \mathrm{H}]<0.3$, which reaches $0.03 \mathrm{dex}$ at $[\mathrm{Fe} / \mathrm{H}] \sim 0.1$. This residual may reveal a real astrophysical scatter, since uncertainties are unlikely 


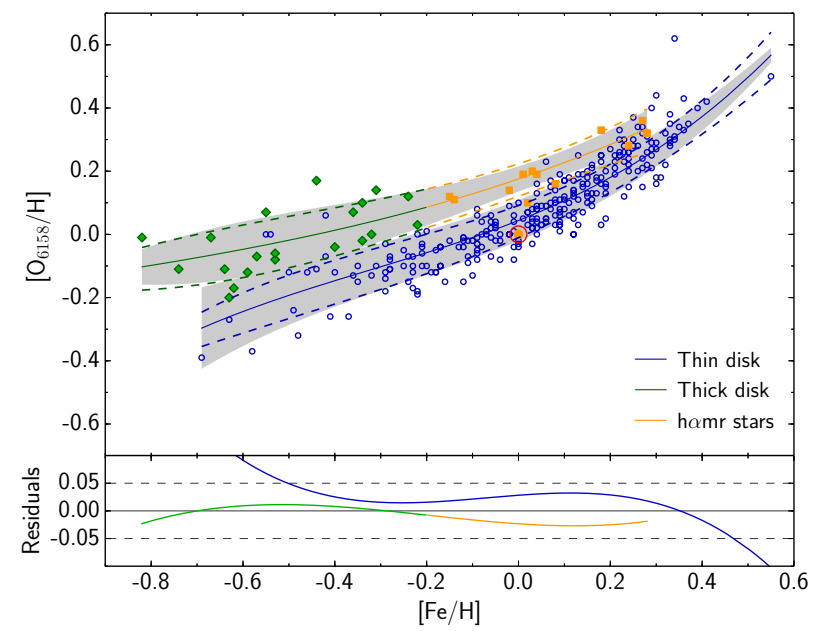

Fig. 14. Upper panel: $[\mathrm{O} / \mathrm{H}]$ vs. $[\mathrm{Fe} / \mathrm{H}]$ for OI $6158 \AA$. Only stars with EW relative error below $25 \%$ are considered. Stars are sepparated in different disk populations according to a chemical criteria. Solid line represent the average oxygen abundance. The $1 \sigma$ level of dispersion around the mean is presented as a shaded area. Dashed line shows the average uncertainties. For plotting purposes the average uncertainties are shown around the mean abundance. Lower panel: difference between the $\pm 1 \sigma$ width of the scatter around the mean and the $\pm 1 \sigma$ width of the uncertainties.

to be underestimated. On the other hand, it can be checked that the thick and thin disk populations appear to be separated at a $1 \sigma$ level, thus confirming the enrichment of $\alpha$-elements in the thick disk. In addition, the enhancement of the h $\alpha \mathrm{mr}$ population is clearly revealed.

\subsection{Comparision with previous studies}

Several studies have addressed the behaviour of oxygen over iron in the thin and thick disk populations. In general, all of them agree that thick disk stars show an enhancement of $\alpha$ elements, being this caused by different ratios of SNII/SNIa in both disks. Some of them found a shallow decline in the $[\mathrm{O} / \mathrm{Fe}]$ trend at high $[\mathrm{Fe} / \mathrm{H}]$ for thin disk stars (e.g. Bensby et al. 2004, 2014; Ramírez et al. 2007; Takeda \& Honda 2005; Petigura \& Marcy 2011), indicating that in the thin disk both SN Ia and SN II contribute at a steady rate to the enrichment of the interstellar medium. In contrast, Ramírez et al. (2013) and Nissen et al. (2002, 2014) results suggest a flattening of $[\mathrm{O} / \mathrm{Fe}]$ at super-solar $[\mathrm{Fe} / \mathrm{H}]$. Our data also suggest that flattening in the $[\mathrm{O} / \mathrm{Fe}]$ ratio may appear at solar metallicities (Fig. 12). The "knee" is inappreciable in the case of [OI], and thus, for the moment we cannot rule out that a change of slope below $[\mathrm{Fe} / \mathrm{H}] \sim 0$ is real. This apparent lack of agreement between both oxygen indicators at high $[\mathrm{Fe} / \mathrm{H}]$ can be attributed either to the scatter in the [OI]6300 data or because some blend may appear at high metallicities near the OI6158 $\AA$ line, leading to an overestimation of the EW. However, although further work needs to be done to study the linelist near $6158 \AA$ spectral line, neither the FWHM or the symmetry of the line seem to suggest the presence of an unknown blend.

In order to study the behaviour of $[\mathrm{O} / \mathrm{Fe}]$ towards the metalpoor regime in Fig. 15 we compare our results of OI6158 $\AA$ with those of Ramírez et al. (2013). These authors derive oxygen abundances from the infrared triplet and correct them for nonLTE effects, but neglect inelastic collisions with hydrogen. We also make a comparison with the results of Bensby et al. (2014) and Nissen et al. (2014), both of them based on a combination of samples for which oxygen was measured using [OI]6300 and the OI IR triplet, and only the OI IR triplet, respectively. Nissen et al. (2014) corrected oxygen abundances for departures from LTE accounting for $\mathrm{H}$ collisions, as described by the classical Drawin formula (Drawin 1968), and scaled by $S_{\mathrm{H}}=0.85$. Bensby et al. (2014) used for this purpose an empirical correction derived by comparing abundances of 60 stars from [OI]6300 and OI774 indicators.

While our results mostly agree with those of Bensby et al. (2014) and Nissen et al. (2014), we find clear disagreement for the metal-poor stars of Ramírez et al. (2013). Figure 15 shows at $[\mathrm{Fe} / \mathrm{H}]<-0.5$ how the $[\mathrm{O} / \mathrm{Fe}]$ rise becomes steeper and we obtain much larger values for $[\mathrm{O} / \mathrm{Fe}]$ than Ramírez et al. (2013). On the other hand, despite the similar slope, our results reach higher $[\mathrm{O} / \mathrm{Fe}]$ ratios than Nissen et al. (2014) and Bensby et al. (2014) at $[\mathrm{Fe} / \mathrm{H}] \sim-0.8 \mathrm{dex}$. Although the most metal-poor stars in our sample have weak OI6158 $\AA$ oxygen lines, with EW between 2 and $4 \mathrm{~m} \AA$, we can safely rule out the possibility that our values are overestimated because of the poor continuum measurements and/or other sources of errors. To illustrate this point, in Fig. 16 we show one of the targets in our sample HD 22879 with one of the highest $[\mathrm{O} / \mathrm{Fe}]=0.81$ and $[\mathrm{Fe} / \mathrm{H}]=-0.82$. The combined spectrum of this star has $S / N=1000$ and our precise measurements of OI6158 $\AA \mathrm{EW}$, which perfectly match the synthetic spectra, clearly rule out an EW weaker than $2.5 \mathrm{~m} \AA$. Thus, our best value for this star is $[\mathrm{O} / \mathrm{Fe}]=0.81$. These high oxygen over iron ratios are also found with [OI] measurements, as an example HD 25704 is a star with $[\mathrm{Fe} / \mathrm{H}]=-0.83$ and $[\mathrm{O} / \mathrm{Fe}]=0.69$ dex. Finally, it is noteworthy to mention that the observed trend towards higher values of $\left[\mathrm{O}_{6158} / \mathrm{H}\right]$ at low temperatures (Fig. 5) do not affect the observed $[\mathrm{O} / \mathrm{Fe}]$ trend since none of the high $[\mathrm{O} / \mathrm{Fe}]$ stars have $T_{\text {eff }}<5600 \mathrm{~K}$.

Given the quality of the observations and measurements presented in these studies, the simplest way to explain the discrepancy with other works rely on the different non-LTE corrections applied to the abundance calculations. Differences with Ramírez et al. (2013) arise because the inelastic collisions with $\mathrm{H}$ atoms were neglected in their OI IR triplet calculations, which would have shifted the trend towards the LTE results. Actually, calculations of non-LTE by Fabbian et al. (2009) taking into account inelastic collisions with neutral oxygen conclude that nonLTE effects presented by these authors were overestimated by $\sim 0.10$ dex at $[\mathrm{Fe} / \mathrm{H}] \sim-1.5 \mathrm{dex}$. On the other hand, the disagreement found between [OI]6300 and OI IR triplet in the Sun lead us to question the non-LTE correction applied by Bensby et al. (2014). Although this correction may be a good first approach to the problem, it would be useful to determine it with more accuracy. Given the current knowledge about departures from the LTE, and the weight of hydrogen collision to bring the system closer to the LTE, Nissen et al. (2014) approach appears to be the most reasonable.

The effects of departures from LTE for $6158 \AA$ transitions have been widely studied in the Sun. Unfortunately, there is not much literature about non-LTE corrections at lower $[\mathrm{Fe} / \mathrm{H}]$ for this spectral line. As a high-excitation line, it is formed in deep layers of the atmospheres, where the source function and line opacity effects work in opposite directions. Thus, the combined effect results in very small non-LTE effects in the Sun $\sim-0.03$ dex (Caffau et al. 2008). However, generally speaking, departures from LTE are more severe towards higher $T_{\text {eff }}$ and $\operatorname{lower} \log (g)$ and $[\mathrm{Fe} / \mathrm{H}]$. Therefore, one would expect larger non-LTE corrections in the low- $[\mathrm{Fe} / \mathrm{H}]$ tail of our 

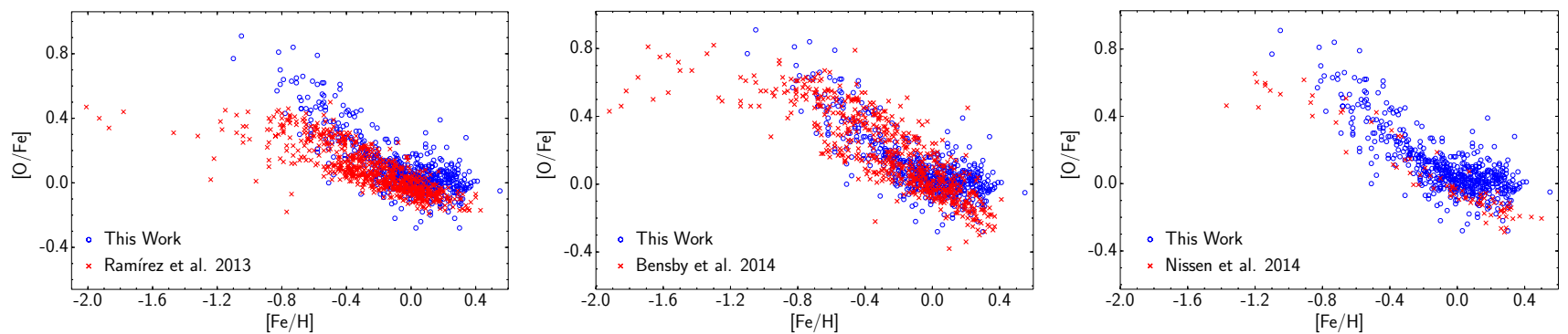

Fig. 15. Comparison of our $\left[\mathrm{O}_{6158} / \mathrm{Fe}\right]$ vs. $[\mathrm{Fe} / \mathrm{H}]$ trends to those derived by Ramírez et al. (2013), Bensby et al. (2014) and Nissen et al. (2014). For comparison purposes, abundance values from Ramírez et al. (2013) and Nissen et al. (2014) have been rescaled to the same solar reference $\left(\log \epsilon(\mathrm{O})_{\odot}=8.71\right)$. Bensby et al. (2014) performed a normalization relative to their solar values on a line-by-line basis. However, individual line abundances are not provided, which makes not possible to rescale to the same solar reference.
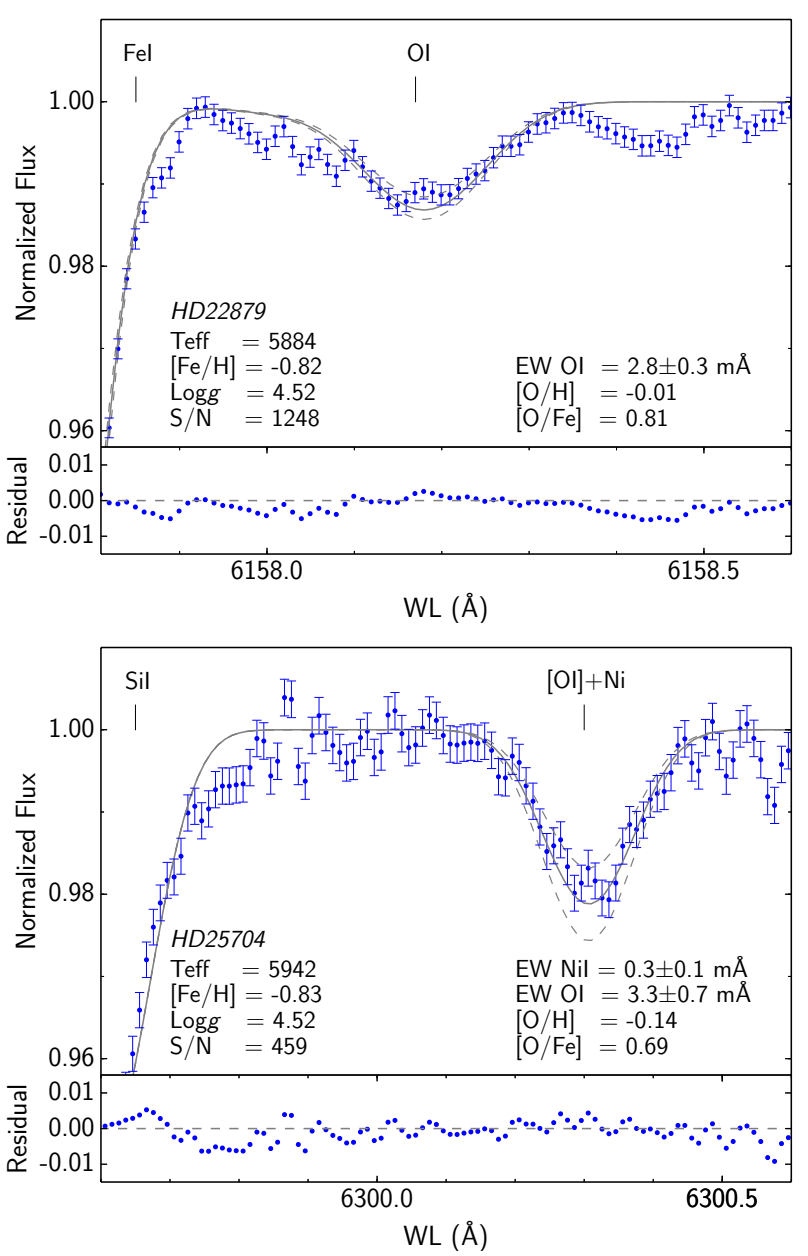

Fig. 16. Spectral synthesis of the oxygen line at $6158 \AA$ in HD 22879 (upper panel) and [OI]6300 in HD 25704 (lower panel). The observed spectra (dots) with the $1 \sigma$ photometric noise (rms) is compared with the synthetic spectra, computed for the corresponding $[\mathrm{Fe} / \mathrm{H}]$ and $[\mathrm{O} / \mathrm{H}]$ abundances obtained from our EW measurements (solid line). Dashed lines represent the synthetic spectra for the $\pm 1 \sigma$ error abundances, which are $\delta[\mathrm{Fe} / \mathrm{H}]= \pm 0.03 \mathrm{dex}, \delta\left[\mathrm{O}_{6158} / \mathrm{H}\right]={ }_{-0.07}^{+0.06}$ dex and $\delta\left[\mathrm{O}_{6300} / \mathrm{H}\right]={ }_{-0.18}^{+0.13}$ dex (propagated from the provided EW error). Also shown are the stellar parameters, nominal $\mathrm{S} / \mathrm{N}$, abundances, EW and residual intensities (observed - predicted).

$[\mathrm{O} / \mathrm{Fe}]$ distribution. Temperature is not expected to increase the effect of non-LTE in our stars, for which effective temperatures are between 5600 to $6200 \mathrm{~K}$ at low metallicity. Sitnova et al. (2013) studied non-LTE effects for oxygen permitted transitions in different stars and for a grid of parameters, taking into account hydrogen collisions $\left(S_{\mathrm{H}}=1\right)$. According to their work, non-LTE corrections are not expected to be larger than -0.05 dex in our sample.

\section{Discussion}

In this article we show that the $[\mathrm{O} / \mathrm{Fe}]$ ratio rises steeply with decreasing metallicity, and reaches 0.8 around $[\mathrm{Fe} / \mathrm{H}]=-1$. Our data are not extended below $[\mathrm{Fe} / \mathrm{H}]=-1$ and it is not clear if this ratio will stay constant or keep rising in halo stars down to $[\mathrm{Fe} / \mathrm{H}]<-3$. Obviously both oxygen lines employed in this work will disappear in halo dwarfs since their EWs will be less than $1 \mathrm{~m} \AA$. Thus, there is no chance to extend this study to $\mathrm{F}$ type metal-poor halo dwarfs. Finally, we note that despite considerable observational effort the trend of the $[\mathrm{O} / \mathrm{Fe}]$ ratio in the halo is still uncertain. However, most of the studies (Israelian et al. 2001; Takeda 2003; Fulbright \& Johnson 2003) suggest that main-sequence dwarfs provide more reliable and consistent abundances than giants.

A steep rise of the $[\mathrm{O} / \mathrm{Fe}]$ ratio and high values above 0.7 found in the present analysis can hardly be explained in the framework of classical models of galactic chemical evolution. We consider, for example, the models proposed by Chiappini et al. (2001). This model considers two main accretion episodes for the formation of the Galaxy, the first forming the halo and bulge in a short timescale and the second one forming the thin disk. They propose a short halo formation timescale of about 0.8 Gyr. At a certain epoch (coinciding with the halo-disk transition) core-collapse $\mathrm{SNe}$, responsible for the production of $\mathrm{O}$, stopped exploding while Fe, produced by the long-living SNe Ia, continued to be produced. Star formation must have ceased for a period that cannot be longer than 1 Gyr. The two-infall model fits the observed metallicity distribution of the G-dwarfs by assuming a long timescale for the thin-disk formation. These models have been calculated assuming a constant plateau at $[\mathrm{Fe} / \mathrm{H}]<-1$ for all $\alpha$-elements including oxygen, for which they propose $[\mathrm{O} / \mathrm{Fe}]=0.5$ at $[\mathrm{Fe} / \mathrm{H}]=-1$. These models predict a light increase in $[\mathrm{O} / \mathrm{Fe}]$ towards lower $[\mathrm{Fe} / \mathrm{H}]$ reaching $[\mathrm{O} / \mathrm{Fe}] \sim 0.4$ at $[\mathrm{Fe} / \mathrm{H}]=-1$. However, some authors (e.g. Boesgaard et al. 2011) have already reported that $\alpha$-elements do not show a plateau at $[\mathrm{Fe} / \mathrm{H}]<-1$. Moreover, the steep rise of $[\mathrm{O} / \mathrm{Fe}]$ does not necessarily imply the same behaviour for other $\alpha$-elements (e.g. Ramaty et al. 2000). The different trend for oxygen compared to the other $\alpha$-elements at $[\mathrm{Fe} / \mathrm{H}]>0$ can also be explained by the fact that oxygen is only produced in Type II $\mathrm{SN}$. The levelling out of the $[\alpha / \mathrm{Fe}]$ trend for other $\alpha$ elements is clearly expected since they also have small contributions from Type Ia SN. Metallicity dependent oxygen yields will make the 
overall picture more complete and may provide additional clues to explain these observations (Prantzos et al. 1994).

Any model for the thick disk origin should account for several observational facts. The first one is the old age of thick disk stars and the temporal gap (1-2 Gyr) with respect to the oldest thin disk stars. Then, the tight relationship found for several abundance ratios in the thick disk. And finally, the variation of the abundance ratios with metallicity. It will be an interesting challenge for these models to explain observations of $\alpha$-elements from the same HARPS database (Neves et al. 2009; Adibekyan et al. 2012) as well as $[\mathrm{O} / \mathrm{Fe}]$ trend ratio presented here since they come from the same homogenous sample.

Different models have been proposed to explain observational characteristics of the thick disk. It is possible that thick disks have been created through accretion of galaxy satellites (Meza et al. 2005; Abadi et al. 2003), where thick-disk stars therefore have an extragalactic origin. It has also been proposed that thick disks were born thick at high redshift from the internal gravitational instabilities in gas-rich clumpy disks (Bournaud et al. 2009; Forbes et al. 2012). Another possibility is that thick disks are created through the heating of pre-existing thin disks with the help of mergers (Quinn et al. 1993; Villalobos \& Helmi 2008), whose rate decreases with decreasing redshift. One could propose that a massive merger will create very intensive star formation at $[\mathrm{Fe} / \mathrm{H}]=-1$ and produce stars with very high $[\mathrm{O} / \mathrm{Fe}]$ ratios.

The thick and thin disks have formed on different timescales. Our data shows that the average ages of thin and thick disks are 4.73 Gyr and 9.20 Gyr, respectively. Details about age determination are provided by Delgado Mena et al. (2014). In fact, all our stars with $[\mathrm{O} / \mathrm{Fe}]>0.6$ belong to the thick disk and are older than 6 Gyr. The Galactic thin disk has not had such an intense star formation history as the thick disk. The shallow decline in the $[\mathrm{O} / \mathrm{Fe}]$ is caused by continuous star formation with no fast initial enrichment from Type II SN.

In the recent past there has been a growing conviction that radial migration can be responsible for the formation of thick disks by bringing out high-velocity-dispersion stars from the inner disk and the bulge (e.g. Schönrich \& Binney 2009). While by now it has been established that because of conservation of vertical action migration does not contribute to disk thickening (Minchev et al. 2012; Martig et al. 2014; Vera-Ciro et al. 2014), radial migration could bring stars with high $[\mathrm{O} / \mathrm{Fe}]$ from inner galaxy to solar neighbourhood (Minchev et al. 2013).

The stellar evolution calculations for massive stars (Woosley \& Weaver 1995; Thielemann et al. 1996) predict that the ejected mass of $(\mathrm{O} / \mathrm{Fe})$ by a massive star is an increasing function of the stellar mass. Thus, an increase of the slope of the IMF during the halo/thick disk transition phase could possibly explain our observations. A very steep linear increase of the $[\mathrm{O} / \mathrm{Fe}]$ in thick disk stars could also be due either to a lower contribution of $\mathrm{Fe}$ from Type II SNe or to a metallicity dependent yield of oxygen (or a combination of these two effects). The change of metallicity on oxygen yield has a different impact depending on the mass range of stars considered (Maeder 1992).

Perhaps the easiest way to explain high $[\mathrm{O} / \mathrm{Fe}]$ ratios in metal-poor stars is by modifying the so called mass-cut parameter in the models of explosive supernova. Present explosive nucleosynthesis calculations for Type II SN are based on induced supernova explosions by either depositing thermal energy or invoking a piston with a given kinetic energy. Induced calculations (lacking self consistency) utilize the constraint of requiring ejected ${ }^{56} \mathrm{Ni}$ masses from the innermost explosive Si burning layers in agreement with supernova light curves being powered by the decay chain ${ }^{56} \mathrm{Ni}_{-}{ }^{56} \mathrm{Co}-{ }^{56} \mathrm{Fe}$. This can also serve as guidance to the supernova mechanism with mass cuts based on ${ }^{56} \mathrm{Ni}$ in the ejecta. It means that the position of a mass cut can define the amount of $\mathrm{Fe}$ ejected from the supernova as well as the $[\mathrm{O} / \mathrm{Fe}]$ ratio in the ejecta (Woosley \& Weaver 1995; Thielemann et al. 1996).

\section{Summary and conclusions}

We present a uniform study of oxygen abundances in a large sample of F- and G-type dwarfs from the HARPS database. A homogeneous set of the atmospheric parameters was adopted, and two independent analyses for different indicators were performed. We provide the first accurate and homogeneous comparison of the OI6158 $\AA$ and [OI]6300 $\AA$ lines of oxygen.

Oxygen is one of the most controversial elements owing to the problems related to inconsistent abundances derived from different spectral lines. In this work we have investigated the behaviour of the lines at $6158 \AA$ and $6300 \AA$. Our measurements suggest that abundances derived from both oxygen lines are consistent within their respective error bars. However, we have found that for spectra with a very high $\mathrm{S} / \mathrm{N}$ these values are no longer compatible within errors. Mainly, the abundance derived from the OI6158 $\AA$ line is 0.072 dex higher than that derived from the $[\mathrm{OI}] 6300 \AA$ A line. A similar difference has been previously reported for the Sun (Caffau et al. 2008). Thus, our results indicate that the disagreement is not unique, and that there must be some physical explanation for this fact.

For F-type stars with temperatures higher than $6200 \mathrm{~K}$, the use of OI6158 $\AA$ is recommended. The EWs of this line are larger than those of the [OI] 6300. Thus, the errors due to the placement of continuum are lower for OI 6158. This line can also be useful in the super-solar metallicity regime where the Ni 6300 line may contribute up to $50 \%$ of the feature at $6300 \AA$. In addition, the uncertainty of $\log (g f)$ for $\mathrm{Ni}$ is non-negligible; therefore, errors in the measurement of [OI] $6300 \AA$ could be significant. In contrast, at low temperatures and metallicity below solar values, OI6158 A becomes weak and yields more uncertain values of oxygen abundances. In any case, both lines can be used in solar analogues if $S / N>300$, allowing us to measure EWs as small as $2 \mathrm{~m} \AA$. With this quality of data, the OI6158 line also represents a good alternative when the [OI]6300 $\AA$ line is severely blended with telluric features. It is always good to measure both lines whenever possible in order to check their consistency.

We found that the abundances derived from the OI6158 line suffer from much smaller dispersion when considering the trends $[\mathrm{O} / \mathrm{H}]$ and $[\mathrm{O} / \mathrm{Fe}]$ vs. $[\mathrm{Fe} / \mathrm{H}]$, in contrast with those derived from the $[\mathrm{OI}] 6300 \AA$ line. The scatter found in the trends for a sub-sample of 252 high-precision measurements of OI6158 $\AA$ is comparable to that of $\mathrm{Mg}$, derived using three spectral lines (Adibekyan et al. 2012). In addition, our results suggest that the $[\mathrm{O} / \mathrm{Fe}]$ ratio reaches a constant value at supersolar metallicities, in contrast with some previous results that reported a shallow decline. A similar trend is seen in the results of Ramírez et al. (2013) and Nissen et al. (2014).

Although there is no clear boundary between oxygen abundances in the thin and thick disks, the stars studied in this article show that some bifurcation might exist at $[\mathrm{Fe} / \mathrm{H}]<-0.3 \mathrm{dex}$, as found with the high-precision sub-sample of OI6158 A. Our results also indicate a clear enhancement of oxygen abundances 
in the thick disk stars, as has been widely reported for other $\alpha$-elements.

Acknowledgements. We thank the anonymous referee for his/her thorough review and suggestions, which significantly contributed to improving the quality of the publication. We are also grateful to Dr. E. Pancino, Dr. C. Allende Prieto and Dr. A. del Pino for fruitful comments and discussion regarding the uncertainties calculations and statistical analysis. S.B. is grateful for financial support from the Spanish Ministry project MICINN AYA2011-29060. This work was also supported by the European Research Council/European Community under the FP7 through Starting Grant agreement number 239953. E.D.M., V.Zh.A. and S.G.S. are supported by grants SFRH/BPD/76606/2011, SFRH/BPD/70574/2010 and SFRH/BPD/47611/2008 from FCT (Portugal), respectively. N.C.S. also acknowledges the support in the form of an Investigador FCT contract funded by FCT/MCTES (Portugal) and POPH/FSE (EC). This work has made use of TOPCAT ${ }^{2}$.

\section{Appendix A: Statistical approach to the determination of chemical abundances}

It is well known that the relation between the EW and the abundance of a spectral line is non-linear. If we assume that the EW has a normal probability distribution, a non-linear transformation will yield an asymmetric abundance distribution. The most obvious consequence of this fact is the asymmetric abundance errors that one can find in any abundance analysis. However, these asymmetries are negligible for strong spectral lines. The present analysis is based on weak lines of oxygen, whose EW is smaller than $5 \mathrm{~m} \AA$ in more than $50 \%$ of the stars. Moreover, EW confidence intervals are not preserved under this non-linear transformation, which means that abundance errors derived from an LTE analysis does not correspond to the same tolerance region. Aiming to provide statistically meaningful uncertainties, we carry out a detailed statistical error analysis.

If we assume a normal probability distribution for the EW, truncated at zero, we can obtain the abundance distribution as (Barlow 2003):

$P([\mathrm{X} / \mathrm{H}])=\frac{G_{T}(E W)}{|\mathrm{d}[\mathrm{X} / \mathrm{H}] / \mathrm{d} E W|}$

where $G_{T}(E W)$ is the adopted distribution for the $\mathrm{EW}$ with mean equal to the measured EW and standard deviation calculated as the final $1 \sigma$ uncertainty of the EW. A simple relation between abundance and EW is provided by the curve of growth. Considering a single spectrum line, we can write:

$m \log (E W)=[\mathrm{X} / \mathrm{H}]+c$

where $m$ and $c$ are constant coefficients. For each star we can find these coefficients by evaluating two values of EW, and deriving their abundances following the standard LTE analysis. The probability density function of the abundance will be:

$P([\mathrm{X} / \mathrm{H}])=\frac{\ln 10}{m \sigma \sqrt{2 \pi}} 10^{\frac{[\mathrm{X} / \mathrm{H}]-c}{m}} G_{T}\left(10^{\frac{[\mathrm{X} / \mathrm{H}]-c}{m}}\right)$.

We can test the correctness of this model by randomly generating a set of EW values following a normal distribution, truncated at zero. The assumed abundance distribution should be able to reproduce the distribution of the random EWs, transformed into abundance using the curve of growth. This test is shown in Fig. A.1.

Because of the non-linearity of the transformation, this distribution is remarkably asymmetric. Several problems will arise

\footnotetext{
2 http://www.starlink.ac.uk/topcat/
}

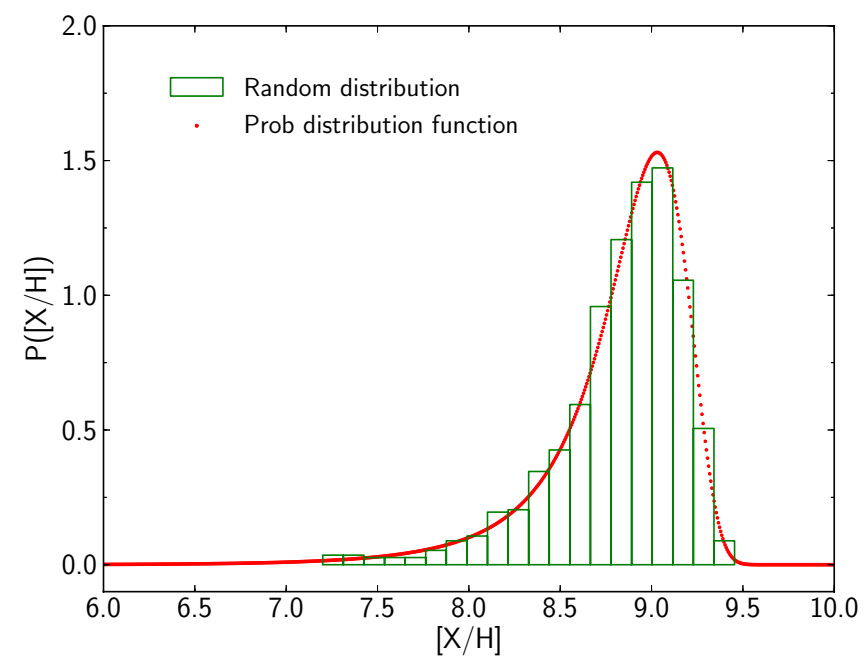

Fig. A.1. Abundance distribution probability for a large asymmetry case. Red dots represent the analytically derived function. Green bars represent the distribution of the abundance calculated with the curve of growth for a set of random EW values that follow a truncated Gaussian distribution.

from this fact, such as the asymmetry of the abundance errors or the biased result towards the high tail which is obtained from the LTE abundance analysis.

Ideally, the normal distribution of the EW is not truncated, and the measured EW of the spectral line corresponds to the mean, median and mode, as expected for normal distributions. Under monotonic transformations the median is preserved and therefore the abundance from the LTE analysis will be the median of the distribution, acceptably quoted as a result, but no longer the mean or mode. However, when we address the measurement of small lines, the normal distribution is truncated at zero as a result of the negative values of EW. In this situation the measured EW is only the mode of the distribution (not the median), and thus its transformation will not yield the median of the abundance probability distribution: the abundance derived in the LTE analysis will not only be biased towards the left tail of the distribution, but will also be smaller than the median. In this case, it becomes especially useful to calculate the probability distribution in order to provide statistically consistent results. Abundances derived from the LTE analysis have been used in the present work; however, we provide in Tables A.2 and A.1 the statistical results. Figure A. 2 shows the deviation of the LTE abundances from the median value of the statistical distribution. It is shown that for EWs larger than $8 \mathrm{~m} \AA$, regardless of the $\mathrm{S} / \mathrm{N}$, the result from the LTE analysis coincide with the median of the abundance statistical probability distribution.

Abundance uncertainties are often calculated separately and added together in quadrature (positive and negative contributions independently). Nevertheless, this procedure is not justified mathematically (Barlow 2003). Throughout this paper we provided the errors in EW, which are symmetric. However, the uncertainties in abundance should be calculated using the probability distribution. The errors are calculated as the abundance values where the probabilities are 0.159 and 0.841 for the negative and positive errors, respectively. These abundance intervals would enclose the $\pm 1 \sigma$ region around the median, in a normal distribution. We present in Fig. A.3 the median abundance with the corresponding uncertainties. Tables A.2 and A.1 show abundance results from this statistical analysis for the OI6158 $\AA$ and [OI]6300 Å lines, respectively. 

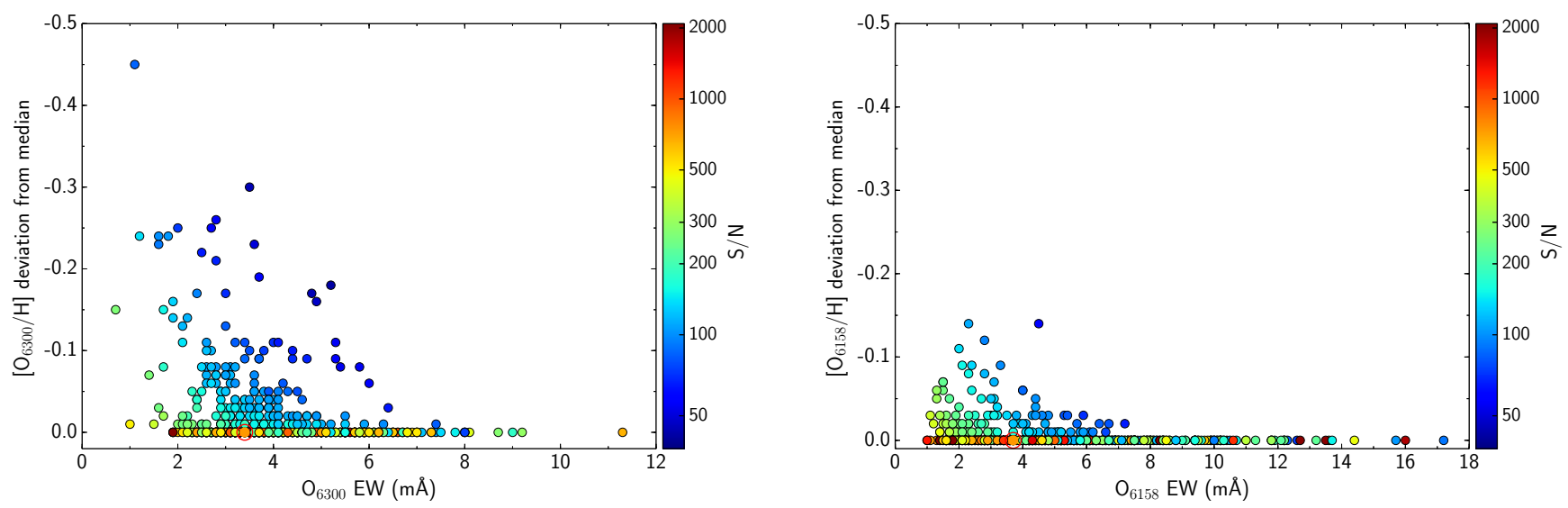

Fig. A.2. Deviation of the LTE abundances from the median value of the statistical probability distribution. Dependence on the nominal signal-tonoise is shown in colours.
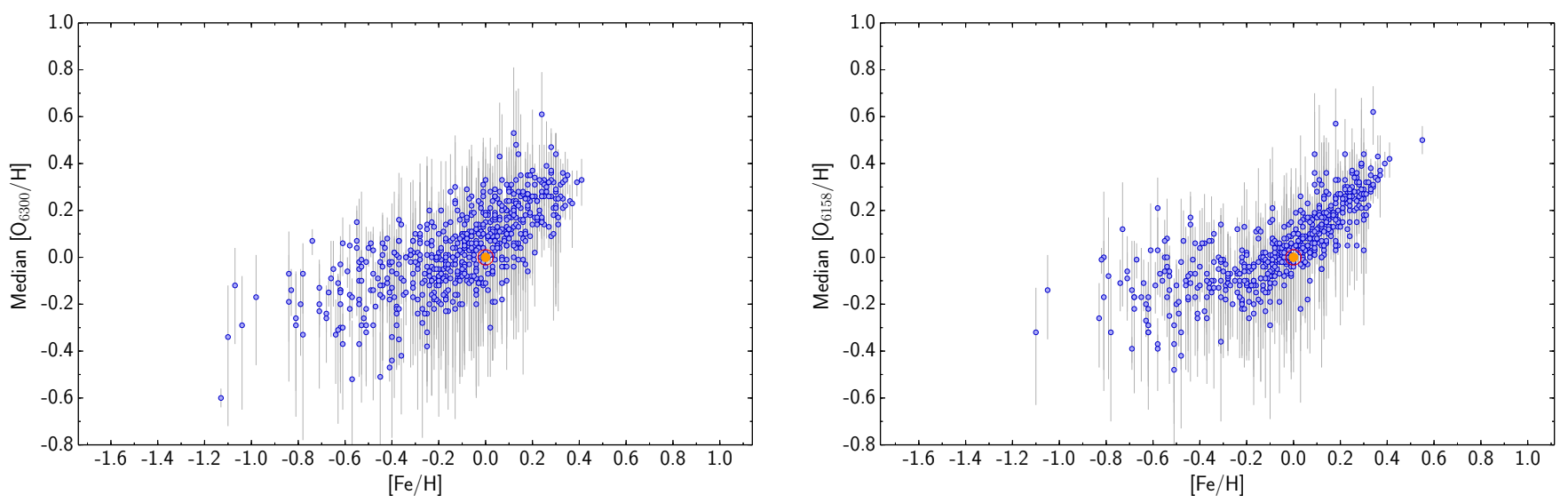

Fig. A.3. $[\mathrm{O} / \mathrm{H}]$ vs. $[\mathrm{Fe} / \mathrm{H}]$ for both oxygen abundance indicators, where $[\mathrm{O} / \mathrm{H}]$ is taken as the median of the statistical distribution. The yellow dot corresponds to the solar abundances. $[\mathrm{O} / \mathrm{H}]$ absolute error bars are over-plotted.

Table A.1. Statistical oxygen abundances from the OI 6158 A line.

\begin{tabular}{lccccccc}
\hline \hline Star & $E W_{\text {OI }}(\mathrm{m} \AA)$ & $\delta E W_{\text {OI }}(\mathrm{m} \AA)$ & {$[\mathrm{O} / \mathrm{H}]_{\mathrm{OI}}$} & Median $[\mathrm{O} / \mathrm{H}]_{\mathrm{OI}}$ & $\sigma_{\mathrm{OI}}$ & $+\delta[\mathrm{O} / \mathrm{H}]_{\mathrm{OI}}$ & $-\delta[\mathrm{O} / \mathrm{H}]_{\mathrm{OI}}$ \\
\hline Sun & 3.7 & 0.3 & 0.00 & 0.00 & 0.04 & 0.04 & -0.05 \\
HD 70889 & 6.9 & 0.5 & 0.10 & 0.10 & 0.04 & 0.04 & -0.04 \\
HD 21161 & 7.5 & 2.8 & 0.18 & 0.18 & 0.27 & 0.18 & -0.26 \\
HD 37226 & 7.4 & 0.8 & -0.01 & -0.01 & 0.06 & 0.06 & -0.06 \\
$\ldots$ & $\ldots$ & $\ldots$ & $\ldots$ & $\ldots$ & $\ldots$ & $\ldots$ & $\ldots$ \\
\hline
\end{tabular}

Notes. The full table is available at the CDS.

Table A.2. Statistical oxygen abundances from the [OI] $6300 \AA$ Aine.

\begin{tabular}{lccccccc}
\hline \hline Star & $E W_{[\mathrm{OI}]}(\mathrm{m \AA})$ & $\delta E W_{[\mathrm{OI}]}(\mathrm{m \AA})$ & {$[\mathrm{O} / \mathrm{H}]_{[\mathrm{OI}]}$} & Median $[\mathrm{O} / \mathrm{H}]_{[\mathrm{OI}]}$ & $\sigma_{[\mathrm{OI}]}$ & $+\delta[\mathrm{O} / \mathrm{H}]_{[\mathrm{O}]}$ & $-\delta[\mathrm{O} / \mathrm{H}]_{[\mathrm{OI}]}$ \\
\hline Sun & 3.4 & 0.3 & 0.00 & 0.00 & 0.04 & 0.04 & -0.05 \\
HD 48611 & 1.9 & 0.8 & -0.42 & -0.42 & 0.26 & 0.16 & -0.25 \\
HD 70889 & 3.3 & 0.4 & 0.10 & 0.10 & 0.05 & 0.05 & -0.06 \\
HD 21161 & 5.9 & 2.9 & 0.23 & 0.24 & 0.29 & 0.17 & -0.28 \\
$\ldots$ & $\ldots$ & $\ldots$ & $\ldots$ & $\ldots$ & $\ldots$ & $\ldots$ & $\ldots$ \\
\hline
\end{tabular}

Notes. The full table is available at the CDS. 


\section{References}

Abadi, M. G., Navarro, J. F., Steinmetz, M., \& Eke, V. R. 2003, ApJ, 597, 21 Adibekyan, V. Z., Santos, N. C., Sousa, S. G., \& Israelian, G. 2011, A\&A, 535, L11

Adibekyan, V. Z., Sousa, S. G., Santos, N. C., et al. 2012, A\&A, 545, A32 Adibekyan, V. Z., Figueira, P., Santos, N. C., et al. 2013, A\&A, 554, A44 Allende Prieto, C., Lambert, D. L., \& Asplund, M. 2001, ApJ, 556, L63 Anders, E., \& Grevesse, N. 1989, Geochim. Cosmochim. Acta, 53, 197

Asplund, M., Grevesse, N., Sauval, A. J., Allende Prieto, C., \& Kiselman, D. 2004, A\&A, 417, 751

Asplund, M., Grevesse, N., Sauval, A. J., \& Scott, P. 2009, ARA\&A, 47, 481

Ayres, T. R. 2008, ApJ, 686, 731

Barlow, R. 2003 [arXiv: astro-ph/0306138]

Bensby, T., Feltzing, S., \& Lundström, I. 2004, A\&A, 415, 155

Bensby, T., Feltzing, S., \& Oey, M. S. 2014, A\&A, 562, A71

Boesgaard, A. M., King, J. R., Deliyannis, C. P., \& Vogt, S. S. 1999, AJ, 117, 492

Boesgaard, A. M., Rich, J. A., Levesque, E. M., \& Bowler, B. P. 2011, ApJ, 743, 140

Bohlin, R. C., Jenkins, E. B., Spitzer, Jr., L., et al. 1983, ApJS, 51, 277

Bournaud, F., Elmegreen, B. G., \& Martig, M. 2009, ApJ, 707, L1

Caffau, E., Ludwig, H.-G., Steffen, M., et al. 2008, A\&A, 488, 1031

Cayrel, R. 1988, in The Impact of Very High S/N Spectroscopy on Stellar

Physics, eds. G. Cayrel de Strobel, \& M. Spite, IAU Symp., 132, 345

Chiappini, C., Matteucci, F., \& Romano, D. 2001, ApJ, 554, 1044

Delgado Mena, E., Israelian, G., González Hernández, J. I., et al. 2010, ApJ, 725, 2349

Delgado Mena, E., Israelian, G., González Hernández, J. I., et al. 2014, A\&A, 562, A92

Drawin, H.-W. 1968, Z. Phys., 211, 404

Ecuvillon, A., Israelian, G., Santos, N. C., et al. 2006, A\&A, 445, 633

Fabbian, D., Asplund, M., Barklem, P. S., Carlsson, M., \& Kiselman, D. 2009, A\&A, 500, 1221

Forbes, J., Krumholz, M., \& Burkert, A. 2012, ApJ, 754, 48

Fuhrmann, K. 1998, A\&A, 338, 161

Fulbright, J. P., \& Johnson, J. A. 2003, ApJ, 595, 1154

Gray, D. F. 1992, The observation and analysis of stellar photospheres (Cambridge: Cambridge University Press)

Helmi, A., Williams, M., Freeman, K. C., Bland-Hawthorn, J., \& De Silva, G. 2014, ApJ, 791, 135

Israelian, G., García López, R. J., \& Rebolo, R. 1998, ApJ, 507, 805

Israelian, G., Rebolo, R., García López, R. J., et al. 2001, ApJ, 551, 833

Israelian, G., Shchukina, N., Rebolo, R., et al. 2004, A\&A, 419, 1095

Johansson, S., Litzén, U., Lundberg, H., \& Zhang, Z. 2003, ApJ, 584, L107

Kurucz, R. 1993, ATLAS9 Stellar Atmosphere Programs and $2 \mathrm{~km} \mathrm{~s}^{-1}$ grid. Kurucz CD-ROM, 13 (Cambridge, Mass.: Smithsonian Astrophysical Observatory)

Kurucz, R. L., Furenlid, I., Brault, J., \& Testerman, L. 1984, Solar flux atlas from 296 to $1300 \mathrm{~nm}$, National Solar Observatory, Atlas $\mathrm{N}^{\circ} 1$ (Sunspot, New Mexico)
Lambert, D. L. 1978, MNRAS, 182, 249

Lo Curto, G., Mayor, M., Benz, W., et al. 2010, A\&A, 512, A48

Maeder, A. 1992, A\&A, 264, 105

Maeder, A. 2009, Physics, Formation and Evolution of Rotating Stars (Heidelberg: Springer Berlin)

Martig, M., Minchev, I., \& Flynn, C. 2014, MNRAS, 442, 2474

Mayor, M., Pepe, F., Queloz, D., et al. 2003, The Messenger, 114, 20

Meza, A., Navarro, J. F., Abadi, M. G., \& Steinmetz, M. 2005, MNRAS, 359, 93

Minchev, I., Famaey, B., Quillen, A. C., et al. 2012, A\&A, 548, A127

Minchev, I., Chiappini, C., \& Martig, M. 2013, A\&A, 558, A9

Neves, V., Santos, N. C., Sousa, S. G., Correia, A. C. M., \& Israelian, G. 2009, A\&A, 497, 563

Nissen, P. E., Primas, F., Asplund, M., \& Lambert, D. L. 2002, A\&A, 390, 235

Nissen, P. E., Chen, Y. Q., Carigi, L., Schuster, W. J., \& Zhao, G. 2014, A\&A, 568, A25

Petigura, E. A., \& Marcy, G. W. 2011, ApJ, 735, 41

Prantzos, N., Vangioni-Flam, E., \& Chauveau, S. 1994, A\&A, 285, 132

Prochaska, J. X., Naumov, S. O., Carney, B. W., McWilliam, A., \& Wolfe, A. M. 2000, AJ, 120, 2513

Quinn, P. J., Hernquist, L., \& Fullagar, D. P. 1993, ApJ, 403, 74

Ramaty, R., Scully, S. T., Lingenfelter, R. E., \& Kozlovsky, B. 2000, ApJ, 534, 747

Ramírez, I., Allende Prieto, C., \& Lambert, D. L. 2007, A\&A, 465, 271

Ramírez, I., Allende Prieto, C., \& Lambert, D. L. 2013, ApJ, 764, 78

Santos, N. C., Mayor, M., Bonfils, X., et al. 2011, A\&A, 526, A112

Santos, N. C., Sousa, S. G., Mortier, A., et al. 2013, A\&A, 556, A150

Schönrich, R., \& Binney, J. 2009, MNRAS, 399, 1145

Sitnova, T. M., Mashonkina, L. I., \& Ryabchikova, T. A. 2013, Astron. Lett., 39 126

Sneden, C. A. 1973, Ph.D. Thesis, The University of Texas, Austin

Socas-Navarro, H., \& Norton, A. A. 2007, ApJ, 660, L153

Sousa, S. G., Santos, N. C., Mayor, M., et al. 2008, A\&A, 487, 373

Sousa, S. G., Santos, N. C., Israelian, G., et al. 2011a, A\&A, 526, A99

Sousa, S. G., Santos, N. C., Israelian, G., Mayor, M., \& Udry, S. 2011b, A\&A, 533, A141

Stasińska, G., Prantzos, N., Meynet, G., et al. 2012, Oxygen in the Universe, EAS Pub. Ser., 54

Takeda, Y. 2003, A\&A, 402, 343

Takeda, Y., \& Honda, S. 2005, PASJ, 57, 65

Teske, J. K., Schuler, S. C., Cunha, K., Smith, V. V., \& Griffith, C. A. 2013, ApJ, 768, L12

Thielemann, F.-K., Nomoto, K., \& Hashimoto, M.-A. 1996, ApJ, 460, 408

Thielemann, F.-K., Argast, D., Brachwitz, F., et al. 2002, Ap\&SS, 281, 25

Tsantaki, M., Sousa, S. G., Adibekyan, V. Z., et al. 2013, A\&A, 555, A150

Valenti, J. A., \& Fischer, D. A. 2005, ApJS, 159, 141

Vera-Ciro, C., D’Onghia, E., Navarro, J., \& Abadi, M. 2014, ApJ, 794, 173

Villalobos, Á., \& Helmi, A. 2008, MNRAS, 391, 1806

Wheeler, J. C., Sneden, C., \& Truran, Jr., J. W. 1989, ARA\&A, 27, 279

Woosley, S. E., \& Weaver, T. A. 1995, ApJS, 101, 181 